\title{
Efficient schemes to compute diffusive barrier crossing rates
}

\author{
By M. J. RUIZ-MONTERO†, D. FRENKEL $\ddagger$ and J. J. BREY† \\ $\dagger$ Física Teórica. Facultad de Física. Apdo. de Correos 1065. 41080 Sevilla, Spain \\ \# FOM Institute for Atomic and Molecular Physics, Kruislaan 407, 1098 SJ \\ Amsterdam, The Netherlands
}

(Received 3 September 1996; revised version accepted 28 October 1996)

\begin{abstract}
The formulation of the classical barrier-crossing problem is reviewed in the context of numerical simulations, with the focus on barrier crossing problems where the reaction coordinate depends in a non-trivial way on the Cartesian coordinates of many particles. Often it is convenient to measure the barrier height using constrained dynamics. Such a calculation requires a knowledge of the Jacobian for the coordinate transformation between Cartesian and generalized ('reaction') coordinates, and it is shown that the calculation of this Jacobian can be simplified. The conventional expression for the crossing rate is found to become computationally inefficient when the barrier crossing is diffusive. An alternative formulation of the barrier-crossing rate is given that leads to much better statistical accuracy in the computed crossing rates.
\end{abstract}

\section{Introduction}

Activated processes play an important role in many different areas of (chemical) physics (for a review, see $[1,2])$. During the last twenty years, much progress has been made in deriving for the rate of activated processes statistical mechanical expressions that are convenient to use in numerical simulations (examples are ion association reactions [3] diffusion in solids [4] or crystal nucleation from the melt [5]). Although much of this work is well known, the focus is usually on relatively simple reactive crossings. The aim of the present paper is to discuss some of the technical problems connected with the numerical implementation of the conventional expressions for rate processes in the case of complex (many-body) reaction coordinates and diffusive barrier crossings. To this end, we provide first a coherent description of the theory of activated processes in classical many-body systems, in the context of numerical simulations. In this review we borrow heavily from the ideas of Bennett [4] Chandler [6] and Ciccotti et al. [3] However, our presentation of the theoretical framework is somewhat different and contains some aspects that, to the best of our knowledge, are new. Subsequently, we continue to discuss how the existing techniques can be modified to deal more efficiently with the diffusive barrier crossing problems.

Two concepts play a crucial role in the theory of activated processes: the first is the reaction coordinate $q$ that connects the initial and final states of the system, and the second is the barrier that separates them. The existence of a reaction coordinate means that we can characterize the macroscopic state of the system by a single quantity $q$ that will be in general a function of the configuration of the whole system, i.e., $q=q\left(\mathbf{r}_{1}, \ldots, \mathbf{r}_{N}\right)$. For activated processes, there is a region between the initial and final values of the reaction coordinate where a system in equilibrium is unlikely to be found. We loosely define this region as the 'barrier' separating the initial and final states. In the simplest case, this barrier would be a region of high potential energy, but in general the barrier will correspond to a region of high free energy.

The Bennett-Chandler theory of rate processes is distinct from the more phenomenological theory of Kramers [7] The Kramers theory considers a prototypical activated process, namely the diffusion of a particle over a one-dimensional external potential energy barrier. The influence of the remaining degrees of freedom is then accounted for by an effective friction coefficient. In principle, there is no reason to expect such a description to be valid in general activated processes, especially when the reaction coordinate is a global one. Moreover, even if we assume the Kramers picture to be valid, determining the expression for the 'friction coefficient' in $q$ space is non-trivial.

Before we proceed, let us briefly review why special simulation techniques are required to compute the rate of activated processes. Since the spontaneous crossing of a high barrier is a rare event, the probability of observing such a transition may be negligibly small, even during a very long run. As a consequence, it is not feasible to measure the barrier-crossing rate with good statistical accuracy in a conventional simulation. The solution to this problem $[4,6,8]$ is to generate only 
those trajectories in phase space that are likely to cross the barrier and use the information gathered in these simulations to compute the rate of spontaneous barrier crossing. The most common approach is to consider only trajectories that cross the top of the barrier. However, there is a considerable degree of freedom in the selection of the initial constraint. Clearly, the computed crossing rate should not depend on the sampling scheme that we use.

\section{Response function}

Let us consider a system of $N$ particles having $f$ degrees of freedom. The set of generalized coordinates describing the configuration of the system will be denoted by $q \equiv\left\{q_{1}, \ldots, q_{f}\right\}$, and a point in the associated phase space by $x \equiv\{q, p\}$. The system can be in two macroscopic states, $\mathrm{A}$ and $\mathrm{B}$, and we assume that the coordinate $q_{1}$ can be considered as a reaction coordinate, i.e., a coordinate whose value characterizes the macroscopic state of the system. The Landau free energy as a function of $q_{1}$ has the form shown in figure 1, and we denote the position of the maximum of the barrier by $q_{1}^{*} \cdot q_{1 \mathrm{~A}}$ is the minimum of the free energy corresponding to the macroscopic state $\mathrm{A}$ and $q_{1 \mathrm{~B}}$ is that corresponding to state B. To quantify the state of the system, we define characteristic functions $n_{\mathrm{A}}$ and $n_{\mathrm{B}}$ that measure whether the system is in state A (B). Usually, $n_{\mathrm{A}}$ and $n_{\mathrm{B}}$ are defined as follows:

$$
\begin{aligned}
& n_{\mathrm{A}}=\theta\left(q_{1}^{*}-q_{1}\right), \\
& n_{\mathrm{B}}=\theta\left(q_{1}-q_{1}^{*}\right),
\end{aligned}
$$

where $\theta$ is the Heaviside step function. Of course,

$$
n_{\mathrm{A}}+n_{\mathrm{B}}=1 \text {. }
$$

Moreover,

$$
\begin{gathered}
n_{\mathrm{A}}^{2}=n_{\mathrm{A}}, \\
n_{\mathrm{A}} n_{\mathrm{B}}=0 .
\end{gathered}
$$

However, for what follows the important thing is that equations (4) and (5) hold on average, i.e. $\left\langle n_{\mathrm{A}}^{2}\right\rangle \approx\left\langle n_{\mathrm{A}}\right\rangle$, and $\left\langle n_{\mathrm{A}} n_{\mathrm{B}}\right\rangle \approx 0$. Hence, at a later stage, we are free to choose other characteristic functions for $n_{\mathrm{A}}$ and $n_{\mathrm{B}}$, as long as they differ from the definitions above only in regions of configuration space that contribute negligibly to equilibrium averages. In particular, we are free to choose other functional forms for $n_{\mathrm{A}}$ and $n_{\mathrm{B}}$ at the top of the barrier.

The time evolution of the distribution function $\rho(x, t)$ is given by the Liouville operator, $\mathscr{L}$,

or

$$
\frac{\partial \rho(x, t)}{\partial t}=-\mathscr{L} \rho(x, t)
$$

$$
\rho(x, t)=\mathrm{e}^{-t \mathscr{S}} \rho(x, 0)
$$

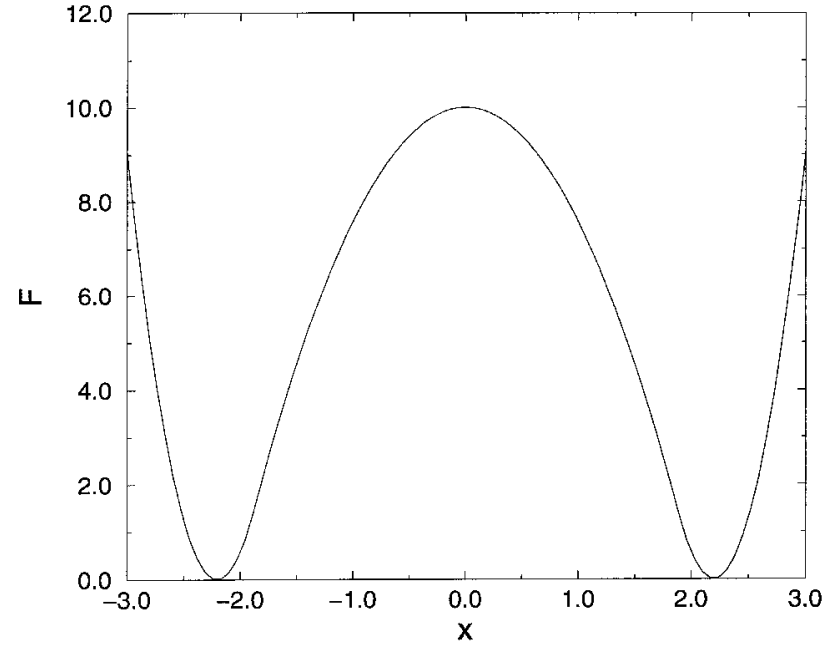

Figure 1. Potential barrier for a typical activated process. In particular, this corresponds to the case discussed in section 9 where the reaction coordinate is the $x$ coordinate of a particle in a Lennard-Jones fluid. The barrier is given by a harmonic potential $U=U_{0}-0 \cdot 5 w^{2} q_{1}^{2}$ with $U_{0}=10 k_{\mathrm{B}} T$ and $w=2 \cdot 2$.

For an arbitrary function $g(x, t)$ it is

$$
g(x, t)=\mathrm{e}^{t \mathscr{B}} g(x, 0) .
$$

Averages with $\rho(x, t)$ can be written as

$$
\langle g(t)\rangle=\int \mathrm{d} x g(x) \rho(x, t)=\int \mathrm{d} x g(x, t) \rho(x, 0),
$$

and for the particular case of the equilibrium distribution;

$$
\langle g\rangle_{\mathrm{eq}}=\int \mathrm{d} x g(x) \rho_{\mathrm{eq}}(x) .
$$

The probability of finding the system in state A at time $t$ will be given by

$$
\begin{aligned}
P_{\mathrm{A}}(t)=\left\langle n_{\mathrm{A}}(t)\right\rangle & =\int \mathrm{d} x n_{\mathrm{A}} \rho(x, t) \\
& =\int \mathrm{d} x \theta\left(q_{1}^{*}-q_{1}\right) \rho(x, t) .
\end{aligned}
$$

We are interested in studying the response function $\phi(t)$ defined as

$$
\phi(t)=\frac{\Delta P_{\mathrm{A}}(t)}{\Delta P_{\mathrm{A}}(0)},
$$

where $\Delta P_{\mathrm{A}}(t)$ is the derivation of $P_{\mathrm{A}}(t)$ from its equilibrium value,

$$
\Delta P_{\mathrm{A}}(t)=P_{\mathrm{A}}(t)-P_{\mathrm{A}, \mathrm{eq}}=\left\langle\Delta n_{\mathrm{A}}(t)\right\rangle
$$

with

$$
\Delta n_{\mathrm{A}}=n_{\mathrm{A}}-\left\langle n_{\mathrm{A}}\right\rangle_{\mathrm{eq}} .
$$

The function $\phi(t)$ gives the approach to equilibrium of the probability of finding the system in state A, subject 
to an initial condition that remains to be specified. To study the behaviour of $\Delta P_{\mathrm{A}}(t)$ it is convenient to take the Laplace transform in equation (6)

$$
\begin{gathered}
z \hat{\rho}(x, z)-\rho(x, 0)=-\mathscr{L} \hat{\rho}(x, z), \\
\hat{\rho}(x, z)=(z+\mathscr{L})^{-1} \rho(x, 0) .
\end{gathered}
$$

Substitution of this result in the expression of $\Delta P_{\mathrm{A}}(t)$ leads to

$$
\begin{aligned}
\Delta \hat{P}_{\mathrm{A}}(z) & =\int \mathrm{d} x \Delta n_{\mathrm{A}} \hat{\rho}(x, z) \\
& =\int \mathrm{d} x \Delta n_{\mathrm{A}}(z+\mathscr{L})^{-1} \rho(x, 0) .
\end{aligned}
$$

Let us assume now an initial condition of the form

$$
\rho(x, 0)=\rho_{\mathrm{eq}}(x) \chi\left(q_{1}\right),
$$

where $\chi$ is a generic non-negative function dependent only on the reaction coordinate $q_{1}$. Normalization of $\rho(x, 0)$ implies

$$
\int \mathrm{d} x \rho_{\mathrm{eq}}(x) \chi\left(q_{1}\right)=1 .
$$

Introducing equation (18) into (17),

$$
\begin{aligned}
\Delta \hat{P}_{\mathrm{A}}(z) & =\int \mathrm{d} x \Delta n_{\mathrm{A}}(z+\mathscr{B})^{-1} \rho_{\mathrm{eq}}(x) \chi\left(q_{1}\right) \\
& =\int \mathrm{d} x \rho_{\mathrm{eq}}(x) \Delta n_{\mathrm{A}}(z+\mathscr{B})^{-1} \chi\left(q_{1}\right) .
\end{aligned}
$$

Therefore,

$$
\hat{\phi}(z)=\frac{\Delta \hat{P}_{\mathrm{A}}(z)}{\Delta P_{\mathrm{A}}(0)}=\frac{\left\langle\Delta n_{\mathrm{A}}(z+\mathscr{L})^{-1} \chi\left(q_{1}\right)\right\rangle_{\mathrm{eq}}}{\left\langle\Delta n_{\mathrm{A}} \chi\right\rangle_{\mathrm{eq}}},
$$

and taking inverse Laplace transform we obtain

$$
\phi(t)=\frac{\left\langle\Delta n_{\mathrm{A}} \mathrm{e}^{-t \mathscr{S}} \chi\left(q_{1}\right)\right\rangle_{\mathrm{eq}}}{\left\langle\Delta n_{\mathrm{A}} \chi\right\rangle_{\mathrm{eq}}} .
$$

Using the properties of the Liouville operator this can be written as

$$
\phi(t)=\frac{\left\langle\Delta n_{\mathrm{A}} \mathrm{e}^{t \mathscr{S}} \chi\left(q_{1}\right)\right\rangle_{\mathrm{eq}}}{\left\langle\Delta n_{\mathrm{A}} \chi\right\rangle_{\mathrm{eq}}}=\frac{\left\langle\Delta n_{\mathrm{A}}(0) \Delta \chi(t)\right\rangle_{\mathrm{eq}}}{\left\langle\Delta n_{\mathrm{A}} \Delta \chi\right\rangle_{\mathrm{eq}}} .
$$

\section{Exponential relaxation}

Let us suppose now that, after the initial transitory period, $\phi(t)$ decays exponentially, i.e.,

$$
\phi(t)=\mathrm{e}^{-(t / \tau)} .
$$

At this stage, the exponential character of the relaxation function is a hypothesis that remains to be verified in every specific case. In fact, there are examples where this assumption is not justified [9] In each particular pro- blem, we will have to find our whether an exponential law is or is not valid for describing the relaxation of the system. The time $\tau$ characterizes the transition of the system from state A to state B and, for activated processes with a high barrier, we can expect it to be very large.

The relaxation time $\tau$ often is expressed in terms of the Laplace transform of the relaxation function at $z=0$ :

$$
\hat{\phi}(z=0)=\int_{0}^{\infty} \mathrm{d} t \phi(t) \equiv \tau .
$$

Using equation (21) we obtain

$$
\tau=\lim _{z \rightarrow 0} \frac{\left\langle\Delta n_{\mathrm{A}}(z+\mathscr{B})^{-1} \chi\right\rangle_{\mathrm{eq}}}{\left\langle\Delta n_{\mathrm{A}} \Delta \chi\right\rangle_{\mathrm{eq}}} .
$$

We are interested in deriving a microscopic expression for the relaxation time. Differentiating the exponential relaxation function yields

$$
\frac{\mathrm{d} \phi}{\mathrm{d} t}=-\frac{1}{\tau} \mathrm{e}^{-(t / \tau)}
$$

On the other hand, from equation (23) we obtain

$$
\frac{\mathrm{d} \phi}{\mathrm{d} t}=\frac{\left\langle\Delta n_{\mathrm{A}} \mathrm{e}^{t \mathscr{B}} \mathscr{B} \Delta \chi\right\rangle_{\mathrm{eq}}}{\left\langle\Delta n_{\mathrm{A}} \Delta \chi\right\rangle_{\mathrm{eq}}} .
$$

From the definition of the Liouville operator it follows that

$$
\mathscr{B} \Delta \chi=-\{\mathscr{H}, \Delta \chi\}=\frac{\partial \mathscr{H}}{\partial p_{1}} \frac{\partial \Delta \chi}{\partial q_{1}}=\dot{q}_{1} \chi\left(q_{1}\right),
$$

where $\mathscr{H}$ is the Hamiltonian of the system and the brackets (braces) denote Poisson brackets. We then obtain

$$
\frac{\mathrm{d} \phi}{\mathrm{d} t}=\frac{\left\langle\Delta n_{\mathrm{A}} \dot{q}_{1}(t) \chi\left\lceil q_{1}(t)\right]_{\mathrm{eq}}\right.}{\left\langle\Delta n_{\mathrm{A}} \Delta \chi\right\rangle_{\mathrm{eq}}}=-\frac{\left\langle\dot{q}_{1} \chi\left(q_{1}\right) n_{\mathrm{A}}(t)\right\rangle_{\mathrm{eq}}}{\left\langle\Delta n_{\mathrm{A}} \Delta \chi\right\rangle_{\mathrm{eq}}},
$$

where we have used the properties of the Liouville operator and we have also taken into account that the equilibrium average of $\dot{q}_{1} \chi\left(q_{1}\right)$ is zero, as it is an odd function of the momenta.

By equating expressions (30) and (27) one obtains

$$
\frac{1}{\tau} \mathrm{e}^{-(t / \tau)}=\frac{\left\langle\dot{q}_{1} \chi\left(q_{1}\right) n_{\mathrm{A}}(t)\right\rangle_{\mathrm{eq}}}{\left\langle\Delta n_{\mathrm{A}} \Delta \chi\right\rangle_{\mathrm{eq}}} .
$$

This equation shows that the relaxation of the system cannot be exponential at $t=0$ because although the LHS of the previous expression is equal to $-1 / \tau$ at $t=0$, the RHS is zero, as it is the equilibrium average of an odd function of the momenta. Therefore, the exponential law can never be valid at exactly $t=0$. Let us now suppose that we are in a time region where $t \ll \tau$. In that case, $\mathrm{e}^{-t / \tau} \approx 1$ and 


$$
\tau^{-1}=\frac{\left\langle\dot{q}_{1} \chi\left(q_{1}\right) n_{\mathrm{A}}(t)\right\rangle_{\mathrm{eq}}}{\left\langle\Delta n_{\mathrm{A}} \Delta \chi\right\rangle_{\mathrm{eq}}} .
$$

This equation has been obtained under the hypothesis that the relaxation is exponential and it is restricted to times $t \ll \tau$. In the case of activated processes, we can expect $\tau$ to be very large, and it seems reasonable to assume that there is a time window where this condition holds. In fact, in chemical kinetics, it is usually assumed that first-order reactions satisfy this requirement (appendix A). The characteristic relaxation time is related to the rate constant $k_{\mathrm{AB}}$ through

$$
\tau^{-1}=\frac{k_{\mathrm{AB}}}{\left\langle n_{\mathrm{B}}\right\rangle_{\mathrm{eq}}}
$$

where $k_{\mathrm{AB}}$ is the macroscopic transition rate from state A to state B. Comparison of equations (32) and (33) leads to a microscopic expression for $k_{\mathrm{AB}}$ :

$$
\begin{aligned}
M(t) & \equiv \frac{\left\langle n_{\mathrm{B}}\right\rangle_{\mathrm{eq}}}{\left\langle\Delta n_{\mathrm{A}} \Delta \chi\right\rangle_{\mathrm{eq}}}\left\langle\dot{q}_{1} \chi\left(q_{1}\right) n_{\mathrm{A}}(t)\right\rangle_{\mathrm{eq}} \\
& =k_{\mathrm{AB}} .
\end{aligned}
$$

We note that although $k_{\mathrm{AB}}$ is a time independent quantity, the time correlation function $M(t)$ appearing in the first line does depend explicitly on time. Therefore, equation (34) is valid only if and when $M(t)$ reaches a plateau value, after an initial transitory period. The assumption that the phenomenological rate equation is valid implies that the relaxation function is exponential and should remain very close to one, during the initial transitory regime.

To proceed, a specific form for the function $\chi\left(q_{1}\right)$ is needed. The most common choice is [6]

$$
\chi\left(q_{1}\right)=\alpha n_{\mathrm{A}} .
$$

$\alpha$ is a constant that is determined by the normalization condition:

$$
\begin{gathered}
\int \mathrm{d} x \rho_{\mathrm{eq}}(x) \mathrm{\alpha} n_{\mathrm{A}}=1, \\
\alpha=\frac{1}{\left\langle n_{\mathrm{A}}\right\rangle_{\mathrm{eq}}} .
\end{gathered}
$$

Substitution of equation (35) in (34) gives

$$
k_{\mathrm{AB}}=\frac{\left\langle\dot{q}_{1} n_{\mathrm{B}} n_{\mathrm{B}}(t)\right\rangle_{\mathrm{eq}}}{\left\langle n_{\mathrm{A}}\right\rangle_{\mathrm{eq}}} \equiv M(t),
$$

where we have used

$$
\left\langle\Delta n_{\mathrm{A}} \Delta n_{\mathrm{A}}\right\rangle_{\mathrm{eq}}=\left\langle n_{\mathrm{A}}\right\rangle_{\mathrm{eq}}\left\langle n_{\mathrm{B}}\right\rangle_{\mathrm{eq}},
$$

and $n_{\mathrm{A}}(t)=1-n_{\mathrm{B}}(t)$. Again, equation (37) is valid only if $M(t)$ reaches a plateau. In the remainder of this section, and in sections IV-VII, we consider the specific case that the characteristic function is the Heaviside function. We then have

$$
k_{\mathrm{AB}}=\frac{\left\langle\dot{q}_{1} \delta\left(q_{1}-q_{1}^{*}\right) \theta\left(q_{1}(t)-q_{1}^{*}\right)\right\rangle_{\mathrm{eq}}}{\left\langle n_{\mathrm{A}}\right\rangle_{\mathrm{eq}}} \equiv M(t) .
$$

We can now quantify the condition for this plateau to exist. Taking the time derivative of equation (39) we obtain

$$
\frac{\mathrm{d} k_{\mathrm{AB}}}{\mathrm{d} t}=\frac{\left\langle\dot{q}_{1} \delta\left(q_{1}-q_{1}^{*}\right) \dot{q}_{1}(t) \delta\left(q_{1}(t)-q_{1}^{*}\right)\right\rangle_{\mathrm{eq}}}{\left\langle n_{\mathrm{A}}\right\rangle_{\mathrm{eq}}} .
$$

In the plateau region, $\mathrm{d} k_{\mathrm{AB}} / \mathrm{d} t$ should be negligible. Therefore, if a trajectory re-crosses the barrier at time $t$ its velocity should be uncorrelated to the velocity at time $t=0$.

An alternative expression for $k_{\mathrm{AB}}$ is

$$
k_{\mathrm{AB}}=\frac{\left\langle\dot{q}_{1} \delta\left(q_{1}-q_{1}^{*}\right) n_{\mathrm{A}}(-t) n_{\mathrm{B}}(t)\right\rangle_{\mathrm{eq}}}{\left\langle n_{\mathrm{A}}\right\rangle_{\mathrm{eq}}} .
$$

The equivalence of this expression to equation (37) can be shown by using $n_{\mathrm{A}}(-t)=1-n_{\mathrm{B}}(-t)$, and taking into account that, due to the properties of the Liouville operator, $\left\langle\dot{q}_{1} \delta\left(q_{1}-q_{1}^{*}\right) n_{\mathrm{B}}(-t) n_{\mathrm{B}}(t)\right\rangle_{\mathrm{eq}}=0$. Equation (41) shows that only those configurations that are in state $\mathrm{A}$ at time $-t$ and in state $\mathrm{B}$ at time $t$ contribute to the transition rate.

Equations (39) and (41) have a form that is similar to that proposed by Miller [10] for the rate of a chemical reaction:

$$
k_{\mathrm{AB}} \propto\left\langle\dot{q}_{1} \delta\left(q_{1}-q_{1}^{*}\right) R(q, p)\right\rangle_{\mathrm{eq}},
$$

where $R(q, p)$ is an (unspecified) function that measures the 'reactivity' of the trajectory that passes through a given point $(q, p)$ in phase space; $R(q, p)$ is one if the trajectory is reactive and zero otherwise. Clearly, as long as the labelling 'reactive' or 'non-reactive' is unambiguous, $R(p, q)$ is conserved along the trajectory. The problem with $R$ is that its definition depends on the time-window within which we assume that the barrier crossing should take place. If we compare the expression for the rate given in equation (41) with equation (42) we see that a possible definition of $R(p, q)$ would be

$$
R=n_{\mathrm{A}}(-t) n_{\mathrm{B}}(t) \text {. }
$$

In this definition of $R$, a trajectory is considered to be reactive if the system was in state A at time - $t$ and is in state $\mathrm{B}$ at time $t$. Clearly, this labelling of trajectories is valid only for times $t^{\prime}$ such that $-t \leqslant t^{\prime} \leqslant t$.

Equation (39) is meaningful only if the computed rate constant does not depend on the precise location of the dividing surface $q_{1}^{*}$, provided that $q_{1}^{*}$ is in the barrier region. In appendix $\mathrm{B}$ we show the conditions under which $k_{\mathrm{AB}}$ can be expected to be independent of $q_{1}^{*}$. 


\section{Transition rate}

Let us next consider the behaviour of equation (39) in the limit $t \rightarrow 0^{+}$. In that case we find that

$$
\lim _{t \rightarrow 0^{+}} M(t)=\frac{\left\langle\dot{q}_{1} \delta\left(q_{1}-q_{1}^{*}\right) \theta\left(\dot{q}_{1}\right)\right\rangle_{\mathrm{eq}}}{\left\langle n_{\mathrm{A}}\right\rangle_{\mathrm{eq}}} .
$$

In this limit, the rate constant approaches the value predicted by transition state theory (TST) [11]

$$
\frac{\left\langle\delta\left(q_{1}-q_{1}^{*}\right)\left|\dot{q}_{1}\right|\right\rangle_{\mathrm{eq}}}{2\left\langle n_{\mathrm{A}}\right\rangle_{\mathrm{eq}}}=k_{\mathrm{AB}}^{\mathrm{TST}} .
$$

The difference between the TST expression for the rate and equation (39) is due to re-crossings at the top of the barrier. If the re-crossing is significant, some trajectories that were initially going from A to B will end up on the A side at time $t$ (giving no contribution to $k_{\mathrm{AB}}$ ) and some that were initially going from B to A will end up on the $\mathrm{B}$ side (giving a negative contribution to $k_{\mathrm{AB}}$ ). As a result, $k_{\mathrm{AB}}$ will be smaller than it was at $t=0^{+}$. It is convenient to define a transmission coefficient $\kappa(0 \leqslant \kappa \leqslant 1)$ that accounts for the corrections to the TST prediction for the crossing rate:

$$
\kappa \equiv \frac{k_{\mathrm{AB}}}{k_{\mathrm{AB}}^{\mathrm{TST}}} .
$$

Having considered the limiting behaviour of $M(t)$ for long $(t \ll \tau)$ and short $\left(t \rightarrow 0^{+}\right)$times, let us finally look at the behaviour of $M(t)$ for $t=0$. Somewhat surprisingly,

$$
M(0)=0 \neq \lim _{t \rightarrow 0^{+}} M(t)
$$

The reason why $M(0)$ vanishes is that $M(0)$ is the equilibrium average of an odd function of the momenta:

$$
M(0) \propto\left\langle\dot{q}_{1} \delta\left(q_{1}-q_{1}^{*}\right) n_{\mathrm{B}}(0)\right\rangle_{\mathrm{eq}}=0 .
$$

This discontinuous behaviour of $M(t)$ at $t=0$ is due to the choice of the Heaviside function as the characteristic function, and must be taken into account if we wish to express $k_{\mathrm{AB}}$ as the integral of a flux autocorrelation function. For $t \ll \tau$, we can write,

$$
k_{\mathrm{AB}}=k_{\mathrm{AB}}^{\mathrm{TST}}+\int_{0^{+}} \mathrm{d} t^{\prime} \frac{\mathrm{d} M\left(t^{\prime}\right)}{\mathrm{d} t^{\prime}} .
$$

As $k_{\mathrm{AB}}^{\mathrm{TST}}$ is always an upper bound to the true rate constant, the integral appearing in the previous expression is always negative.

\section{Constrained dynamics and restricted ensembles}

In many applications of practical interest, the reaction coordinate is a complicated function of the configurational coordinates of the system, and a closed equation for its time evolution is not known. Let us therefore consider how equation (39) can be used in molecular dynamics (MD) simulations to compute the transition rate between two macroscopic states $\mathrm{A}$ and $\mathrm{B}$ that are separated by a high (free) energy barrier. Of course, we assume that we are dealing with a situation where equation (39) applies. In particular, we assume that the relaxation of the population of the macroscopic states is effectively exponential.

If we try to compute the RHS of equation (39) directly by standard MD simulation we face a serious difficulty, since at equilibrium the system remains most of the time in the vicinity of the free energy minima, and configurations corresponding to the top of the barrier are seldom reached. Since these are precisely the initial states for trajectories giving a non-vanishing contribution to the RHS of equation (39), it follows that very long computer simulations will be needed in order to obtain good statistics. In fact, the same applies if one uses equation (23) to measure the relaxation function $\phi(t)$. The system has a very small probability of crossing from one macroscopic state to the other during a typical MD run.

One way of dealing with this problem is to use the constrained dynamics method [8] The basic idea is to consider trajectories in configuration space corresponding to the dynamics of the system under a given constraint, and using the constrained averages to obtain information about the unconstrained equilibrium averages. In our case, the constraint will be that we fix the reaction coordinate at the value corresponding to the top of the free energy barrier, i.e., $q_{1}=q_{1}^{*}$. Of course this restricts the number of configurations allowed for the system. In the remainder of this section we review briefly the relation between averages computed in the constrained and unconstrained systems. We will assume for convenience that there is only one reaction coordinate and that it is the only quantity constrained in the dynamics (for the more general case, see [8]).

Let us consider a system of $N$ particles of mass $m_{i}$ whose configuration can be described by a set of generalized coordinates $q \equiv\left\{q_{\alpha} ; \alpha=1, \ldots, 3 N\right\}$. The set is chosen such that it includes the reaction coordinate $q_{1}$. All the generalized coordinates are assumed to be functions of only the positions of the particles. The Hamiltonian of the system is given by the sum of the kinetic and potential energies, $K$ and $U$, respectively:

$$
\not{H}=K+U .
$$


Using the definition of the generalized momenta,

$$
p_{\alpha}=\frac{\partial L}{\partial \dot{q}_{\alpha}},
$$

where $L=K-U$ is the Lagrangian of the system, the kinetic energy can be expressed in the form [12]

$$
K \equiv \frac{1}{2} \sum_{4}^{K} m_{i} \dot{\mathbf{r}}_{i}^{2}=\frac{1}{2} \sum_{4}^{3 N} \sum_{\alpha}^{3 N}\left(\mathscr{G}^{-1}\right)_{\alpha \beta} p_{\alpha} p_{\beta}=\frac{1}{2} p^{+} G^{-1} p .
$$

Here, we have introduced the generalized mass matrix

$$
\mathscr{G}_{\alpha \beta}=\sum_{\mu}^{N} m_{i} \frac{\partial \mathbf{r}_{i}}{\partial q_{\alpha}} \cdot \frac{\partial \mathbf{r}_{i}}{\partial q_{\beta}}
$$

Clearly, $\mathscr{G}$ is a symmetric matrix. The canonical equilibrium distribution of the system at temperature $T$ is

$$
\rho(q, p)=\frac{\mathrm{e}^{-\beta \mathscr{H}(q, p)}}{\int \mathrm{d} q \mathrm{~d} p \mathrm{e}^{-\beta \mathscr{H}(q, p)}},
$$

with $\beta=\left(k_{\mathrm{B}} T\right)^{-1}$, and $k_{\mathrm{B}}$ the Boltzmann constant. Integration over the generalized momenta yields the configurational distribution,

$$
\rho(q)=\int \mathrm{d} p \rho(q, p)=\frac{|\mathscr{G}(q)|^{1 / 2} \mathrm{e}^{-\beta U(q)}}{\int \mathrm{d} q|\mathscr{G}(q)|^{1 / 2} \mathrm{e}^{-\beta U(q)}}
$$

where we have used

$$
\int \mathrm{d} p \exp \left(-\frac{1}{2} \beta p^{+} \cdot \mathscr{G}^{-1} \cdot p\right)=\left(\frac{2 \pi}{\beta}\right)^{3 N / 2}|\mathscr{I}|^{1 / 2} .
$$

Suppose now that the system evolves with time under the constraint that the generalized coordinate $q_{1}$ is fixed at a value $q_{1}^{*}$. Let us denote the set of all generalized coordinates except $q_{1}$, by $q_{\mathrm{s}}$, and similarly for the momenta. The equilibrium distribution of the ensemble generated under the constraint $q_{1}=q^{*}\left(\right.$ and $\left.\dot{q}_{1}=0\right)$ is

$$
\rho_{\mathrm{c}}\left(q_{\mathrm{s}}, p_{\mathrm{s}} ; q_{1}^{*}\right)=\frac{\exp \left[-\beta \mathscr{H}_{\mathrm{s}}\left(q_{\mathrm{s}}, p_{\mathrm{s}} ; q_{1}^{*}\right)\right]}{\int \mathrm{d} q_{\mathrm{s}} \mathrm{d} p_{\mathrm{s}} \exp \left[-\beta \mathscr{H}_{\mathrm{s}}\left(q_{\mathrm{s}}, p_{\mathrm{s}} ; q_{1}^{*}\right)\right]},
$$

i.e., the canonical distribution associated with the 'constrained' Hamiltonian

$$
\mathscr{H}_{\mathrm{s}}\left(q_{\mathrm{s}}, p_{\mathrm{s}} ; q_{1}^{*}\right)=K_{\mathrm{s}}\left(p_{\mathrm{s}}\right)+U\left(q_{1}^{*}, q_{\mathrm{s}}\right)
$$

with

$$
K_{\mathrm{s}}\left(p_{\mathrm{s}}\right)=\frac{1}{2} p_{\mathrm{s}}^{+} \mathscr{H}_{\mathrm{s}}^{-1} p_{\mathrm{s}}
$$

The matrix $\mathscr{G}_{\mathrm{s}}\left(q_{\mathrm{s}} ; q_{1}^{*}\right)$ is the appropriate inverse mass tensor, analogous to $\mathscr{G}$ defined by equation (51), but restricted to the set $q_{\mathrm{s}}$. It depends parametrically on $q_{1}^{*}$. It follows from equation (55) that the configurational probability distribution in the subspace defined by $q_{1}=q_{1}^{*}$ as given by the restricted ensemble is

$$
\rho_{\mathrm{c}}\left(q_{\mathrm{s}} ; q_{1}^{*}\right)=\frac{\left|\mathscr{G}_{\mathrm{s}}\left(q_{\mathrm{s}} ; q_{1}^{*}\right)\right|^{1 / 2} \mathrm{e}^{-\beta U\left(q_{1}^{*}, q_{\mathrm{s}}\right)}}{\int \mathrm{d} q_{\mathrm{s}}\left|\mathscr{G}_{\mathrm{s}}\left(q_{\mathrm{s}} ; q_{1}^{*}\right)\right|^{1 / 2} \mathrm{e}^{-\beta U\left(q_{1}^{*}, q_{\mathrm{s}}\right)}} .
$$

Next, we must relate the above distribution to the probability density of finding the system in configuration $\left(q_{1}^{*}, q_{\mathrm{s}}\right)$ of the unconstrained system. This probability density is given by equation (53):

$$
\rho\left(q_{1}^{*}, q_{\mathrm{s}}\right)=\frac{\left|\mathscr{G}\left(q_{1}^{*}, q_{\mathrm{s}}\right)\right|^{1 / 2} \mathrm{e}^{-\beta U\left(q_{1}^{*}, q_{\mathrm{s}}\right)}}{\int \mathrm{d} q|\mathscr{G}(q)|^{1 / 2} \mathrm{e}^{-\beta U(q)}} .
$$

Comparison of equations (58) and (59) yields

$$
\rho\left(q_{1}^{*}, q_{\mathrm{s}}\right)=C\left(q_{1}^{*}\right)\left(\frac{\left|\mathscr{G}_{(}\left(q_{1}^{*}, q_{\mathrm{s}}\right)\right|}{\left|\mathscr{G}_{\mathrm{s}}\left(q_{\mathrm{s}} ; q_{1}^{*}\right)\right|}\right)^{1 / 2} \rho_{\mathrm{c}}\left(q_{\mathrm{s}} ; q_{1}^{*}\right),
$$

where we have introduced

$$
C\left(q_{1}^{*}\right)=\frac{\int \mathrm{d} q_{\mathrm{s}}\left|\mathscr{G}_{\mathrm{s}}\left(q_{\mathrm{s}} ; q_{1}^{*}\right)\right|^{1 / 2} \mathrm{e}^{-\beta U\left(q_{1}^{*}, q_{\mathrm{s}}\right)}}{\int \mathrm{d} q|\mathscr{G}(q)|^{1 / 2} \mathrm{e}^{-\beta U(q)}},
$$

which does not depend on $q_{\mathrm{s}}$. As is discussed in detail in [8] (for a brief derivation, see appendix C),

$$
\frac{\left|\mathscr{G}_{\mathrm{s}}\left(q_{\mathrm{s}} ; q_{1}^{*}\right)\right|}{\mathscr{G}\left(q_{1}^{*}, q_{\mathrm{s}}\right) \mid}=|H|
$$

with $H$ defined as

$$
H=\sum_{t}^{N} m_{i}^{-1} \frac{\partial q_{1}}{\partial \mathbf{r}_{i}} \cdot \frac{\partial q_{1}}{\partial \mathbf{r}_{i}}
$$

So, we finally obtained

$$
\rho\left(q_{1}^{*}, q_{\mathrm{s}}\right)=C\left(q_{1}^{*}\right)|H|^{1 / 2} \rho_{\mathrm{c}}\left(q_{\mathrm{s}} ; q_{1}^{*}\right) .
$$

The above equation implies that we can obtain averages of configurational quantities in the unrestricted ensemble from averages computed in the restricted one, except for the constant $C\left(q_{1}^{*}\right)$. More explicitly, for an arbitrary function $A(q)$ it is

$$
\left\langle A(q) \delta\left(q_{1}-q_{1}^{*}\right)\right\rangle=C\left(q_{1}^{*}\right)\left\langle|H|^{-1 / 2} A(q)\right\rangle_{c} .
$$

Here and in the following, the subindex $c$ when placed after pointed brackets denotes averages under the constraint $q_{1}=q_{1}^{*}$. As a consequence of equation (65), ratios of averages in the restricted ensemble can be translated directly into ratios of averages in the unrestricted situation through

$$
\frac{\left\langle A(q) \delta\left(q_{1}-q_{1}^{*}\right)\right\rangle}{\left\langle B(q) \delta\left(q_{1}-q_{1}^{*}\right)\right\rangle}=\frac{\left\langle|H|^{-1 / 2} A(q)\right\rangle_{c}}{\left\langle|H|^{-1 / 2} B(q)\right\rangle_{c}} .
$$

Up to this point we have limited ourselves to configurational properties $A(q)$, i.e., to properties depending only on the coordinates of the system. What happens if $A$ also depends on the momenta? The relationship given by equation (60) holds only for the configurational part of the distribution function. The equilibrium momenta distributions generated by the constrained 
and unconstrained dynamics are different and there is not simply a proportionality relation between the two of them. But this is not a serious difficulty since at equilibrium the unconstrained momenta are distributed according to the Maxwell-Boltzmann law. Therefore, a practical way of proceeding is to write from equation (60)

$$
\begin{aligned}
\rho\left(q_{1}^{*}, q_{\mathrm{s}}, p_{1}, p_{\mathrm{s}}\right) & \equiv \rho\left(q_{1}^{*}, q_{\mathrm{s}}\right) F(p) \\
& =C\left(q_{1}^{*}\right)|H|^{-1 / 2} \rho_{\mathrm{c}}\left(q_{\mathrm{s}}, q_{1}^{*}\right) F(p) .
\end{aligned}
$$

The above expression shows clearly that equation (66) also can be applied to compute the equilibrium average of a general function $A(q, p)$, provided we use constrained dynamics to generate the configurational distribution, but unconstrained dynamics to follow the subsequent time evolution of $A$.

Before closing this section, we emphasize that equation (67) shows how constrained dynamics can be used to compute static equilibrium properties of the constrained system. The same equation can be used also to study the time evolution of the system, subject to the condition that the system at $t=0$ satisfies the constraint. However, in that case the constrained dynamics are used only to generate the initial conditions, and the full (unconstrained-constrained) dynamics should be used to study the subsequent time evolution.

\section{Transition rate}

The relation between restricted and unrestricted averages can be used to facilitate the computation of the rate of activated processes with a high free energy barrier. Equation (39) can be rewritten as

$$
k_{\mathrm{AB}}=P_{0}\left(q_{1}^{*}\right) R(t),
$$

where

$$
P_{0}\left(q_{1}^{*}\right)=\frac{\left\langle\delta\left(q_{1}-q_{1}^{*}\right)\right\rangle}{\left\langle\theta\left(q_{1}^{*}-q_{1}\right)\right\rangle}
$$

and

$$
R(t)=\frac{\left\langle\dot{q}_{1} \delta\left(q_{1}-q_{1}^{*}\right) \theta\left[q_{1}(t)-q_{1}^{*}\right]\right.}{\left\langle\delta\left(q_{1}-q_{1}^{*}\right\rangle\right.} .
$$

$P_{0}$ is the equilibrium probability density of finding the system at the top of the barrier divided by the equilibrium probability of finding it in the reactant side. $R(t)$ is the averaged flux at the top of the barrier multiplied by the probability that the system ends up on the product side at time $t . R(t)$ can be measured directly by using constrained dynamics, as it is the ratio of averages involving delta functions of the reaction coordinate. Application of equation (66) yields

$$
R(t)=\frac{\left\langle|H|^{-1 / 2} \dot{q}_{1} \theta\left[q_{1}(t)-q_{1}^{*}\right)\right]_{c}}{\left\langle|H|^{-1 / 2}\right\rangle_{c}}
$$

Unfortunately, $P_{0}$ cannot be computed directly using constrained dynamics. It can, however, be cast in a form that can be computed using constrained dynamics. But, before showing stress, we stress that $P_{0}$ is a timeindependent equilibrium quantity. It can therefore be computed also using Monte Carlo (umbrella) sampling [13] Let us consider the equilibrium probability density $\rho(q 1)$ of finding the system with a given value $q$ i of the reaction coordinate, i.e.,

$$
\rho(q 1)=\left\langle\delta\left(q_{1}-q_{1}\right)\right\rangle=\int \mathrm{d} q \delta\left(q_{1}-q_{1}\right) \rho(q) .
$$

Obviously,

$$
P_{0}\left(q_{1}^{*}\right)=\frac{\rho\left(q_{1}^{*}\right)}{\int_{-\infty}^{q_{1}^{*}} \mathrm{~d} q_{\uparrow} \rho\left(q_{1}\right)} .
$$

We differentiate $\ln \rho(q)$, using equation (53):

$$
\begin{aligned}
\frac{\partial}{\partial q_{1}} \ln \rho\left(q_{1}\right)= & \frac{1}{\int \mathrm{d} q_{\mathrm{s}}\left|\mathscr{G}\left(q_{1}, q_{\mathrm{s}}\right)\right|^{1 / 2} \mathrm{e}^{-\beta U\left(q_{1}, q_{\mathrm{s}}\right)}} \frac{\partial}{\partial q_{1}} \\
& \times \int \mathrm{d} q_{\mathrm{s}}\left|\mathscr{G}\left(q_{1}, q_{\mathrm{s}}\right)\right|^{1 / 2} \mathrm{e}^{-\beta U\left(q_{1}, q_{\mathrm{s}}\right)} .
\end{aligned}
$$

It is easy to verify that the above expression is equivalent to

$$
\frac{\partial}{\partial q_{\uparrow}} \ln \rho\left(q_{\uparrow}\right)=\frac{\left\langle F\left(q_{\rho}, q_{\mathrm{s}}\right) \delta\left(q_{1}-q_{\mathcal{\rho}}\right)\right\rangle}{\left\langle\delta\left(q_{1}-q_{\uparrow}\right)\right\rangle},
$$

where we have defined

$$
F\left(q_{1}, q_{\mathrm{s}}\right)=\frac{\partial \ln \left|\mathscr{G}\left(q_{1}, q_{\mathrm{s}}\right)\right|^{1 / 2}}{\partial q_{1}}-\beta \frac{\partial U\left(q_{1}, q_{\mathrm{s}}\right)}{\partial q_{1}} .
$$

The RHS of equation (75) can be expressed as a ratio of constrained averages through equation (66),

$$
\frac{\partial}{\partial q_{1}} \ln \rho(q \uparrow)=\frac{\left\langle\left. H\left(q \uparrow, q_{\mathrm{s}}\right)\right|^{-1 / 2} F\left(q \rho, q_{\mathrm{s}}\right)\right\rangle_{\mathrm{c}^{\prime}}}{\left\langle\left|H\left(q_{\uparrow}, q_{\mathrm{s}}\right)\right|^{-1 / 2}\right\rangle_{\mathrm{c}^{\prime}}} .
$$

Here, the subscript $c^{\prime}$ denotes averaging in an ensemble subject to the constraint $q_{1}=q$ r. In order to obtain $\rho\left(q_{1}^{*}\right)$ we must integrate equation (77), starting from some reference value of $q$ s and up to $q_{1}^{*}$. A convenient choice is $q_{1}=q_{1 \mathrm{~A}}$, i.e., the position of the minimum of the free energy corresponding to state A. The value of $\rho\left(q_{1 \mathrm{~A}}\right)$ can be obtained from a direct equilibrium simulation. Then, we arrive at

$$
\ln \frac{\rho\left(q_{1}^{*}\right)}{\rho\left(q_{1 \mathrm{~A}}\right)}=\int_{q_{1 \mathrm{~A}}}^{\rho_{1}^{*}} \mathrm{~d} q_{1} \frac{\left\langle\left|H\left(q 1, q_{\mathrm{s}}\right)\right|^{-1 / 2} F\left(q 1, q_{\mathrm{s}}\right)\right\rangle_{\mathrm{c}^{\prime}}}{\left\langle\left|H\left(q_{1}, q_{\mathrm{s}}\right)\right|^{-1 / 2}\right\rangle_{\mathrm{c}}} .
$$

In the expression for $F\left(q_{1}, q_{\mathrm{s}}\right)$, equation (76), the determinant $|\mathscr{G}|$ can be replaced by the Jacobian $|J|$ of the transformation from the Cartesian coordinates $\mathbf{r}_{i}$ to the 
generalized ones $q_{\alpha}$. In effect, from the definition of $\mathscr{G}$, equation (51), it follows that

$$
\mid\left(J^{-1}\right)^{+} \mathscr{G} J^{-1}=\prod_{l=1}^{N} m_{i}^{3}
$$

which implies

$$
|\mathscr{G}|^{1 / 2}=|J| \prod_{l=1}^{N} m_{i}^{3 / 2}
$$

and

$$
\frac{\partial \ln |\mathscr{G}|^{1 / 2}}{\partial q_{1}}=\frac{\partial \ln |J|}{\partial q_{1}} .
$$

Then we can rewrite equation (76) as

$$
F\left(q_{1}, q_{\mathrm{s}}\right)=\frac{\partial \ln \left|J\left(q_{1}, q_{\mathrm{s}}\right)\right|}{\partial q_{1}}-\beta \frac{\partial U\left(q_{1}, q_{\mathrm{s}}\right)}{\partial q_{1}},
$$

which is the form which appears in the literature [8]

In practical applications of the scheme described above, the choice of the reaction coordinate $q_{1}$ associated with the reaction order parameter is dictated by the physics of the problem. However, there is considerable freedom in the choice of the complementary set of generalized coordinates $q_{\mathrm{s}}$. Of course, the final result for the transition rate does not depend on this choice, but the calculations simplify if an appropriate set of generalized coordinates is considered. To the best of our knowledge, this fact has not been exploited sufficiently. Below, we show how a judicious choice of generalized coordinates leads to a significant simplification of the calculations. Such a simplification becomes particularly important when $q_{1}$ is a global parameter depending on the position of all the particles in the system. In this case, it is convenient to choose the complementary set in such a way that the generalized coordinates are orthogonal:

$$
\nabla q_{\alpha} \cdot \nabla q_{\beta}=\delta_{\alpha \beta}
$$

and

$$
\nabla q_{1} \cdot \nabla q_{\alpha}=0
$$

for $\alpha, \beta>1$. Here, we have introduced the gradient

$$
\nabla \equiv\left(\frac{\partial}{\partial \mathbf{r}_{1}}, \ldots, \frac{\partial}{\partial \mathbf{r}_{N}}\right)
$$

In appendix D we show that equations (83) and (84) imply

$$
|J|=\left|\nabla q_{1}\right|^{-1}
$$

and

$$
\frac{\partial f}{\partial q_{1}}=\frac{1}{\left|\nabla q_{1}\right|^{2}} \nabla f \cdot \nabla q_{1}
$$

where $f$ is an arbitrary function of the positions of the particles. Using these relations, equation (82) becomes

$$
F\left(q_{1}, q_{\mathrm{s}}\right)=-\frac{\left(\nabla\left|\nabla q_{1}\right|\right) \cdot \nabla q_{1}}{\left|\nabla q_{1}\right|^{3}}-\beta \frac{\nabla U \cdot \nabla q_{1}}{\left|\nabla q_{1}\right|^{2}} .
$$

The first term on the RHS vanishes when $q_{1}$ is a linear function of the Cartesian coordinates and then the only contribution comes from the gradient of the potential energy. On the other hand, if $q_{1}$ is a complicated nonlinear function of the positions of the particles, the contribution of this term may be quite significant. The important fact is that equation (88) allows the evaluation of $F$ and, therefore, of the transition rate in a direct way from the expression of the reaction coordinate $q_{1}$, without any need for further specifying the complementary set of generalized coordinates. An application of the method has been reported recently for the case of nucleation of a simple fluid [14] where the reaction coordinate is a complex, nonlinear function of the full configuration of the system.

\section{Drawbacks}

In the previous sections we reviewed the derivation of the standard statistical mechanics expression for the rate of activated processes that is amenable to numerical simulation (equation (39)). However, in numerical simulation it is important to distinguish between expressions that are correct in principle, and those that are correct and computationally efficient. So the question we wish to address is whether equation (39) for the rate constant is optimal from a computational point of view. The question is important because equation (39) has been the starting point for most molecular dynamics simulations of activated processes. However, as we will show below, equation (39) is most useful when it is least needed, i.e., when re-crossing is not important and the transition state theory prediction for the rate is reasonably accurate.

Let us first consider a simple example to illustrate the problem: a square barrier of height $U$ and width $\omega$ that separates two macroscopic states, A and B. When the system is in equilibrium, the two states have the same probability $\rho_{\text {eq }}$. We assume that the motion in the barrier region is diff usive. In that case, the time evolution of the system is governed by the Kramers equation [7]

$$
\frac{\partial x \rho\left(q_{1}, t\right)}{\partial t}=\frac{\partial}{\partial q_{1}}\left[D \beta U\left(q_{1}\right) \rho\left(q_{1}, t\right)\right]+D \frac{\partial^{2} \rho\left(q_{1}, t\right)}{\partial q_{1}^{2}}
$$

where $D$ is the diffusion constant of the system. This equation follows from the continuity equation

$$
\frac{\partial \rho\left(q_{1}, t\right)}{\partial t}=-\frac{\partial}{\partial q_{1}} J\left(q_{1}, t\right)
$$


for $J\left(q_{1}, t\right)$, the probability flux, and the constitutive equation

$$
J\left(q_{1}, t\right)=-D\left[\beta U\left(q_{1}\right) \rho\left(q_{1}, t\right)+\frac{\partial \rho\left(q_{1}, t\right)}{\partial q_{1}}\right] .
$$

From equation (89) it follows that, if the system is in a steady state, the probability distribution at the top of the barrier (where $U^{\prime}=0$ ) is a linear function of the reaction coordinate $q_{1}$,

$$
\rho^{\text {st }}\left(q_{1}\right)=a q_{1}+b \text { if } 0<q_{1}<\omega,
$$

and the flux of probability is constant,

$$
J^{\text {st }}=-a D \text {. }
$$

The constants $a$ and $b$ have to be determined from the boundary conditions. Let us suppose now that initially we increase the probability of state A from its equilibrium value by an amount $\varepsilon \rho_{\mathrm{eq}} / 2$, and decrease the probability of state B by the same amount. If the barrier is high enough, the flux will be very small and the probabilities of states A and B will not change. Alternatively, we can think that the system is in contact with a reservoir and the probabilities of states A and B do not change in time. In this case, the stationary probability distribution at the top of the barrier is

$$
\rho^{\mathrm{st}}\left(q_{1}\right)=\mathrm{e}^{-\beta U} \rho_{\mathrm{eq}}\left[1-\left(q_{1}-\frac{\omega}{2}\right) \frac{\varepsilon}{\omega}\right],
$$

and the flux is

$$
J^{\mathrm{st}}=-D \frac{\varepsilon \rho_{\mathrm{eq}}}{\omega} \mathrm{e}^{-\beta U} \text {. }
$$

As expected, the flux decreases exponentially with the barrier height. It should be stressed that, if the probability at the top of the barrier does not have the form given by equation (94), the flux has not reached its stationary value.

Let us come back now to our expression for the rate. Using the properties of the Liouville operator equation (39) can be rewritten as

$$
k_{\mathrm{AB}} \equiv M(t)=\frac{\left\langle\theta\left[q_{1}^{*}-q_{1}(0)\right] \dot{1}_{1}(t) \delta\left(1 q_{1}(t)-q_{1}^{*}\right)\right\rangle_{\mathrm{eq}}}{\left\langle n_{\mathrm{A}}\right\rangle_{\mathrm{eq}}} .
$$

Apart from a constant factor, $M(t)$ is the flux through the transition state $q_{1}^{*}$ (that we choose, somewhat arbitrarily, to be in the middle of the barrier, i.e., $q_{1}^{*}=\omega / 2$ ), due to a step function probability profile at $t=0$. As this step function differs from the linear profile that corresponds to the steady state, the subsequent flux will depend on time. We are interested in the plateau value of $M(t)$ after the initial transitory regime. The usual assumption is that this transitory regime extends over typical 'molecular' time scales. However, in the present case it is easy to show that the approach of
$M(t)$ to its plateau value can be quite slow. For times $t \ll \omega^{2} / D$ we can combine equations (89) and (91) to yield

$$
\frac{\partial J\left(q_{1}^{*}, t\right)}{\partial t} \approx D \frac{\partial^{2} J\left(q_{1}^{*}, t\right)}{\partial q_{1}^{2}} .
$$

We then find that $J\left(q_{1}^{*}, t\right)$ decays as $1 / t^{1 / 2}$ for times $t \ll \omega^{2} / D$. This means that the approach to the stationary state is rather slow. Moreover, in the case of diffusive barrier crossings, the transmission coefficient $\kappa$ is typically quite small. But, as we will show, such small values of $\kappa$ cannot be measured accurately using equation (39).

The expression for the transition coefficient is

$$
\kappa=\frac{2}{\left\langle\left|\dot{q}_{1}\right|\right\rangle_{\mathrm{eq}}}\left\langle\dot{q}_{1}(0) n_{\mathrm{B}}(t)\right\rangle_{\mathrm{c}} .
$$

In a computer simulation, we put the system initially at $q_{1}^{*}$ and let it evolve. We then compute $n_{\mathrm{B}}(t)$ for times that are long enough for equation (39) to have reached a plateau value. We repeat this procedure for $n$ independent trajectories, and then estimate $\kappa$ as

$$
\kappa_{\mathrm{est}}=\frac{2}{n\left\langle\left|\dot{q}_{1}\right|\right\rangle_{\mathrm{eq}}} \sum^{n}\left[\dot{q}_{1}(0) n_{\mathrm{B}}(t)\right] .
$$

The statistical error in $\kappa_{\text {est }}$ is given by

$$
\sigma_{\kappa}^{2}=\left\langle\left(\kappa_{\text {est }}-\langle\kappa)^{2}\right\rangle\right. \text {. }
$$

Taking into account that the trajectories are uncorrelated and assuming that $\dot{q}_{1}$ and $n_{\mathrm{B}}$ are Gaussian variables [15] we obtain

$$
\left.\sigma_{\kappa}^{2}=\frac{4}{n\left\langle\dot{q}_{1} \mid\right\rangle_{\mathrm{eq}}^{2}}\left\langle\dot{q}_{1}^{2}\right\rangle n_{\mathrm{B}}^{2}\right\rangle+\frac{1}{n} \kappa^{2} .
$$

If the transmission coefficient is very small, the second contribution in the previous expression is negligible, and

Moreover,

$$
\sigma_{\kappa}^{2} \sim \frac{4}{n\left\langle\left|\dot{q}_{1}\right|\right\rangle_{\mathrm{eq}}^{2}}\left\langle\dot{q}_{1}^{2}\right\rangle\left\langle n_{\mathrm{B}}^{2}\right\rangle
$$

$$
4 \frac{\left\langle\dot{q}_{1}^{2}\right\rangle}{\left\langle\left|\dot{q}_{1}\right|\right\rangle_{\mathrm{eq}}^{2}} \sim 1 .
$$

Hence,

$$
\sigma_{\kappa}^{2} \sim \frac{1}{n},
$$

and the relative error is

$$
\frac{\sigma_{\kappa}}{\kappa} \sim \frac{1}{\kappa V_{n}} .
$$

This shows that even for a transmission coefficient of $0 \cdot 1$ we would need to follow about $10^{4}$ trajectories in order to get a $10 \%$ accuracy. 
The obvious question is whether we can do better. The main problem with equation (39) is that we prepare the system in a state that is not close to the steady state situation. In the steady state, the probability profile at the top of the barrier is a linear function of the reaction coordinate, and the flux is very small. Hence, if we set up a perturbation that has the desired shape, rather than a step function, we would eliminate the problem of the slow diffusive approach to the steady state crossing rate. We will use, therefore, equation (34) for the rate, and, instead of choosing a step function for the generic function $\chi\left(q_{1}\right)$, we assume that $\chi$ outside the barrier region behaves like a step function, while inside the barrier region it has the same $q_{1}$ dependence as the steady-state concentration profile:

$$
\chi\left(q_{1}\right)=\alpha f\left(q_{1}\right),
$$

with

$$
f\left(q_{1}\right)=\left\{\begin{array}{cl}
1 & \text { if } q_{1}<0 \\
1-\frac{q_{1}}{\omega} & \text { if } 0<q_{1}<\omega \\
0 & \text { if } q_{1}>\omega .
\end{array}\right.
$$

As the initial perturbation is a linear function of $q_{1}$ at the top of the barrier, the system is immediately prepared in the steady state. The constant $\alpha$ is fixed by the normalization condition

$$
\alpha \approx \frac{1}{\left\langle n_{\mathrm{A}}\right\rangle_{\mathrm{eq}}},
$$

where we have used the fact that $f\left(q_{1}\right)$ differs from a step function only in the barrier region, which contributes negligibly to the integral. For the same reason, we can write

$$
\left\langle\Delta \chi \Delta n_{\mathrm{A}}\right\rangle_{\mathrm{eq}} \approx\left\langle n_{\mathrm{B}}\right\rangle_{\mathrm{eq}}
$$

Introducing these last two results in equation (34), we obtain

$$
k_{\mathrm{AB}}=\frac{1}{\left\langle n_{\mathrm{A}}\right\rangle_{\mathrm{eq}}}\left\langle\dot{q}_{1} f\left(q_{1}\right) n_{\mathrm{A}}(t)\right\rangle_{\mathrm{eq}}
$$

Using the expression for $f\left(q_{1}\right)$,

$$
\left\langle\dot{q}_{1} f\left(q_{1}\right) n_{\mathrm{A}}(t)\right\rangle_{\mathrm{eq}}=-\frac{1}{\omega}\left\langle\dot{q}_{1} \theta\left(q_{1}\right) \theta\left(\omega-q_{1}\right) n_{\mathrm{A}}(t)\right\rangle_{\mathrm{eq}}
$$

As the average $\left\langle\dot{q}_{1} f\left(q_{1}\right) n_{\mathrm{A}}(0)\right\rangle_{\mathrm{eq}}$ vanishes, we need consider only the change in $n_{\mathrm{A}}$ during time $t$ :

$$
\begin{aligned}
n_{\mathrm{A}}(t)-n_{\mathrm{A}}(0) & =\int_{0} \mathrm{~d} t^{\prime} \frac{\mathrm{d} n_{\mathrm{A}}\left(t^{\prime}\right)}{d t^{\prime}} \\
& =-\int_{0} \mathrm{~d} t^{\prime} \dot{q}_{1}\left(t^{\prime}\right) \delta\left(q_{1}\left(t^{\prime}\right)-q_{1}^{*}\right) .
\end{aligned}
$$

$$
\begin{aligned}
& \text { Hence, } \\
& \begin{aligned}
\left\langle\dot{q}_{1} f\left(q_{1}\right) n_{\mathrm{A}}(t)\right\rangle_{\mathrm{eq}}= & \frac{1}{\omega} \int_{0} \mathrm{~d} t^{\prime}\left\langle\dot{q}_{1} \theta\left(q_{1}\right) \theta\left(\omega-q_{1}\right)\right. \\
& \left.\times \dot{q}_{1}\left(t^{\prime}\right) \delta\left(q_{1}\left(t^{\prime}\right)-q_{1}^{*}\right)\right\rangle_{\mathrm{eq}} \\
= & \frac{1}{\omega} \int_{0} \mathrm{~d} t^{\prime}\left\langle\dot{q}_{1} \delta\left(q_{1}-q_{1}^{*}\right) \dot{q}_{1}\left(t^{\prime}\right)\right. \\
& \left.\times \theta\left(q_{1}\left(t^{\prime}\right)\right) \theta\left(\omega-q_{1}\left(t^{\prime}\right)\right)\right\rangle_{\mathrm{eq}} .
\end{aligned}
\end{aligned}
$$

The integrand is the velocity autocorrelation function of a system that is initially at $q_{1}^{*}$ (at the middle of the barrier), multiplied by the probability that it has not left the top of the barrier at time $t^{\prime}$. As the velocity of the system becomes uncorrelated with the initial one after a few collisions (i.e., on a truly 'molecular' time scale), we can expect the velocity autocorrelation function to have decayed to zero before the system leaves the top of the barrier. Then,

$$
\left\langle\dot{q}_{1} f\left(q_{1}\right) n_{\mathrm{A}}(t)\right\rangle_{\mathrm{eq}}=\frac{1}{\omega} \int_{0} \mathrm{~d} t^{\prime}\left\langle\dot{q}_{1}(0) \dot{q}_{1}\left(t^{\prime}\right)\right\rangle_{q_{1}^{*}}\left\langle\delta\left(q_{1}-q_{1}^{*}\right)\right\rangle_{\mathrm{eq}} \text {. }
$$

But if the velocity autocorrelation function decays to zero in a time less than $t$ we can replace the upper limit of the previous integral by infinity. Making use of the Green-Kubo relation

$$
D\left(q_{1}^{*}\right)=\int_{0}^{\infty} \mathrm{d} t^{\prime}\left\langle\dot{q}_{1}(0) \dot{q}_{1}\left(t^{\prime}\right)\right\rangle_{q_{1}^{*}},
$$

where we have used the notation $D\left(q_{1}^{*}\right)$ to indicate that $D$ will, in general, depend on $q_{1}$ :

$$
\left\langle\dot{q}_{1} f\left(q_{1}\right) n_{\mathrm{A}}(t)\right\rangle_{\mathrm{eq}}=\frac{D}{\omega}\left\langle\delta\left(q_{1}-q_{1}^{*}\right)\right\rangle_{\mathrm{eq}},
$$

and the rate is

$$
k_{\mathrm{AB}}=\frac{D}{\omega} P_{0}\left(q_{1}^{*}\right),
$$

which is indeed the correct answer. However, this result is not particularly interesting if it does not yield a higher statistical accuracy of the crossing rate. Now our estimate for $\kappa$ is

$$
\kappa_{\text {est }}=\frac{2}{\omega\left\langle\left|\dot{q}_{1}\right|\right\rangle n} \sum_{t}^{n} \int_{0}^{t} \mathrm{~d} t^{\prime}\left(\dot{q}_{1}(0) \dot{q}_{1}\left(t^{\prime}\right)\right)_{i},
$$

where we must remember that in all of the $n$ trajectories considered the system is initially at the top of the barrier. Following essentially the same reasoning that led to equation (101) we now obtain

$$
\begin{aligned}
\left\langle\left(\Delta \kappa_{\text {est }}\right)^{2}\right\rangle= & \frac{4}{\omega^{2}\left\langle\left|\dot{q}_{1}\right|\right\rangle^{2} n}\left\{\int _ { 0 } \mathrm { d } t ^ { \prime } \int _ { 0 } \mathrm { d } t ^ { \prime \prime \prime } \left\langle\dot{q}_{1}(0) \dot{q}_{1}\left(t^{\prime}\right) \dot{q}_{1}(0)\right.\right. \\
& \left.\left.\times \dot{q}_{1}\left(t^{\prime \prime}\right)\right\rangle_{q_{1}^{*}}\left[\int_{0} \mathrm{~d} t^{\prime}\left\langle\dot{q}_{1}(0) \dot{q}_{1}\left(t^{\prime}\right)\right\rangle_{q_{1}^{*}}\right]^{2}\right\} .
\end{aligned}
$$


If we assume, as before, that $\dot{q}_{1}$ is a Gaussian variable,

$$
\begin{aligned}
\left\langle\left(\Delta \kappa_{\text {est }}\right)^{2}\right\rangle= & \frac{4}{\omega^{2}\left\langle\left|\dot{q}_{1}\right|\right\rangle^{2} n} \\
& \times\left[\left\langle\dot{q}_{1}^{2}\right\rangle t \int_{0} \mathrm{~d} t^{\prime}\left\langle\dot{q}_{1}(0) \dot{q}_{1}\left(t^{\prime}\right)\right\rangle_{q_{1}^{*}}+D^{2}\right], \\
\left\langle\left(\Delta \kappa_{\text {est }}\right)^{2}\right\rangle \sim & \frac{4}{\omega^{2}\left\langle\left|\dot{q}_{1}\right|\right\rangle^{2} n}\left\langle\dot{q}_{1}^{2}\right\rangle D t,
\end{aligned}
$$

where we have used the fact that the second factor in equation (120) is $\kappa^{2} / n$, which is negligible in the diffusive regime, where $\kappa$ is small. Using equation (103) again,

$$
\left\langle\left(\Delta \kappa_{\mathrm{est}}\right)^{2}\right\rangle \sim \frac{D t}{\omega^{2} n}
$$

The relative error in the computation of the transmission coefficient is now

$$
\frac{\left\langle\left(\Delta \kappa_{\mathrm{est}}\right)^{2}\right\rangle^{1 / 2}}{\kappa} \sim \frac{1}{\kappa n^{1 / 2}} \frac{(D t)^{1 / 2}}{\omega} .
$$

We have increased the statistical accuracy by a factor $(D t)^{1 / 2} / \omega$. As $t$ is the time that characterizes the decay of the velocity correlation function, $(D t)^{1 / 2} \sim \lambda_{\mathrm{mf}}, \lambda_{\mathrm{mf}}$ being the mean free path. Hence, $(D t)^{1 / 2} / \omega \sim \lambda_{\mathrm{mf}} / \omega$ which, for barrier widths not of molecular sizes, is very small. Therefore, by a more convenient choice of the characteristic functions defining reactant and products we have increased the statistical accuracy considerably. In fact, it is clear from equation (117) that $\kappa \sim \lambda_{\mathrm{mf}} / \omega$, and the overall effect is

$$
\frac{\left\langle\left(\Delta \kappa_{\mathrm{est}}\right)^{2}\right\rangle^{1 / 2}}{\kappa} \sim \frac{1}{n^{1 / 2}} .
$$

We have improved the statistical accuracy by a factor/ $\kappa$. Clearly, for diffusive barrier crossing problems, there is much to be gained by using a smoother function than the $\theta$ function to measure the properties of the barrier crossing problem.

\section{General case}

Let us consider again the general barrier crossing problem: a system that can be in two states A and B separated by a free energy barrier $F\left(q_{1}\right)$. To construct an efficient numerical scheme, we will again assume that the time evolution of the system is described reasonably well by the Kramers equation:

$$
\frac{\partial \rho\left(q_{1}, t\right)}{\partial t}=-\frac{\partial J\left(q_{1}, t\right)}{\partial q_{1}}
$$

where $J\left(q_{1}, t\right)$ is the probability flux

$$
\begin{aligned}
J\left(q_{1}, t\right) & =-D_{q}\left[\beta F\left(q_{1}\right) \rho\left(q_{1}, t\right)+\frac{\partial \rho\left(q_{1}, t\right)}{\partial q_{1}}\right] \\
& =-D_{q} \mathrm{e}^{-\beta F\left(q_{1}\right)} \frac{\partial}{\partial q_{1}}\left[\mathrm{e}^{\beta F\left(q_{1}\right)} \rho\left(q_{1}, t\right)\right]
\end{aligned}
$$

$D_{q}$ is the diffusive coefficient in $q_{1}$ space. Equation (125) can be written in terms of the operator $\mathscr{D}$ as

$$
\begin{aligned}
\frac{\partial \rho\left(q_{1}, t\right)}{\partial t} & =D_{q} \frac{\partial}{\partial q_{1}}\left[\mathrm{e}^{-\beta F\left(q_{1}\right)} \frac{\partial}{\partial q_{1}}\left(\mathrm{e}^{\beta F\left(q_{1}\right)} \rho\left(q_{1}, t\right)\right)\right] \\
& \equiv \mathscr{D} \rho\left(q_{1}, t\right) .
\end{aligned}
$$

The general solution of the above equation is a linear superposition of the eigenfunctions of $\mathscr{P}$ that satisfies [18]

$$
\mathscr{D}_{\varphi_{n}}\left(q_{1}\right)=-\lambda_{n} \varphi_{n}\left(q_{1}\right) \text { for } n=0,1,2, \ldots
$$

$\lambda_{0}=0$, and, obviously, $\varphi_{0}\left(q_{1}\right)$ is the equilibrium distribution associated with the reaction coordinate. The eigenfunctions $\varphi_{n}$ satisfy the orthogonality condition

$$
\int \mathrm{d} q_{1} \varphi_{0}^{-1}\left(q_{1}\right) \varphi_{n}\left(q_{1}\right) \varphi_{m}\left(q_{1}\right)=\delta_{n, m},
$$

and the completeness relation,

$$
\sum=\frac{\varphi_{n}\left(q_{1}\right) \varphi_{n}(q 1)}{\varphi_{0}\left(q_{1}\right)}=\delta\left(q_{1}-q_{1}\right) .
$$

We are interested in the response function of the system after a generic initial perturbation $\chi\left(q_{1}\right)$,

$$
\phi(t)=\frac{\left\langle\Delta n_{\mathrm{A}}(0) \Delta \chi(t)\right\rangle_{\mathrm{eq}}}{\left\langle\Delta n_{\mathrm{A}} \Delta \chi\right\rangle_{\mathrm{eq}}} .
$$

Expansion of the correlation function in eigenfunctions yields

$$
\phi(t)=\sum c_{n} \mathrm{e}^{-\lambda_{n} t},
$$

and

$$
\begin{aligned}
c_{n}= & \frac{1}{\left\langle\Delta n_{\mathrm{A}} \Delta \chi\right\rangle_{\mathrm{eq}}}\left[\int \mathrm{d} q_{1} \Delta n_{\mathrm{A} \varphi_{n}}\left(q_{1}\right)\right] \\
& \times\left[\int \mathrm{d} q_{1} \Delta \chi\left(q_{1}\right) \varphi_{n}\left(q_{1}\right)\right] .
\end{aligned}
$$

In some specific cases the eigenfunctions and eigenvalues of the operator $\mathscr{P}$ have been worked out $[17,18]$ However, for the present purpose, we do not need to know the full solution. What is important is that, for a sufficiently high barrier, the mode $\varphi_{1}$ decays much more slowly than all higher modes. If we can construct the initial perturbation to be proportional to $\varphi_{1}$, we would suppress the initial transitory behaviour (all $c_{n}$ would be 
zero except $c_{1}$ ), and from the very beginning the system would be in the steady state.

The probability profile that corresponds to $\varphi_{1}$ is constant in region A. In region B it is also constant, but opposite in sign. Only in the barrier region does $\varphi_{1}$ vary rapidly. Let us compute the probability profile of the system when it is in the steady state. If we write the initial perturbation as

$$
\rho\left(q_{1}\right)=\rho_{\mathrm{eq}}\left(q_{1}\right)\left[1+\varepsilon\left(q_{1}\right)\right]
$$

(which means that $\chi=1+\varepsilon\left(q_{1}\right)$ ) we can compute the $q_{1}$ dependence of $\varepsilon\left(q_{1}\right)$ when the system is in the steady state. In that state, the probability flux is constant, independent of $q_{1}$. From equation (126),

$$
J^{\mathrm{st}}=-D_{q} \mathrm{e}^{-\beta F\left(q_{1}\right)} \frac{\partial \varepsilon\left(q_{1}\right)}{\partial q_{1}},
$$

and

$$
\varepsilon\left(q_{1}\right)-\varepsilon_{\mathrm{A}}=-\frac{J^{\mathrm{st}}}{D_{q}} \int_{q_{1 \mathrm{~A}}}^{t_{1}} \mathrm{~d} q_{\mathfrak{1}} \mathrm{e}^{\beta F\left(q_{1}\right)} .
$$

To eliminate $J^{\text {st }}$ from this expression, we impose an absorbing boundary condition at $q_{1 \mathrm{~B}}\left(\varepsilon\left(q_{1 \mathrm{~B}}\right)=0\right)$, leading to

$$
\varepsilon\left(q_{q}\right)=\varepsilon_{\mathrm{A}}\left[1-\frac{\int_{q_{1 \mathrm{~A}}}^{q_{1}} \mathrm{~d} q_{\uparrow} \mathrm{e}^{\beta F\left(q_{1}\right)}}{\left.\int_{q_{1 \mathrm{~A}}}^{q_{1 \mathrm{~B}}} \mathrm{~d} q_{\uparrow} \mathrm{e}^{\beta F\left(q_{1}\right)}\right)}\right] .
$$

If the barrier is high, the value of the integral appearing in the previous equation will be dominated by the maximum of the free energy if the barrier region is inside the integration interval. Therefore, if $q_{1}$ is in the A region, the numerator in equation (136) will be much smaller than the denominator, and $\varepsilon\left(q_{1}\right) / \varepsilon_{\mathrm{A}} \approx 1$. On the other hand, if $q_{1}$ is in the $\mathrm{B}$ region, the numerator and denominator of equation (136) will be approximately equal, and $\varepsilon\left(q_{1}\right) / \varepsilon_{\mathrm{A}} \approx 0$. Hence, $\varepsilon\left(q_{1}\right) / \varepsilon_{\mathrm{A}}$ behaves much like $n_{\mathrm{A}}$, except in the barrier region, where it varies rapidly.

In order to improve the efficiency of the numerical calculation of $k_{\mathrm{AB}}$ we must achieve two things. First of all, we need to suppress, as far as possible, the transient behaviour in the computed rate constant. This is achieved by preparing an initial perturbation that closely resembles the slowest eigenmode $\varphi_{1}$. Second, we need to suppress the statistical noise in $k_{\mathrm{AB}}$ by improving the $\theta$ representation of $n_{\mathrm{A}}$. This is achieved by measuring the progress of the reaction by a function that is proportional to $\varphi_{1}$. In fact, selecting such a characteristic function will also contribute to the suppression of the transient behaviour in $k_{\mathrm{AB}}$.

One of the problems with equation (136) is that usually we do not know exactly the free energy of the system as a function of the order parameter. This problem can be avoided because, in practice, we need to approximate only $F\left(q_{1}\right)$. In what follows, we denote our estimate of $F\left(q_{1}\right)$ by $F_{\text {est }}\left(q_{1}\right)$, and we shall assume that $\varepsilon\left(q_{1}\right)$ in equation (136) is derived from this $F_{\text {est }}\left(q_{1}\right)$. Once we have chosen the form of $\varepsilon\left(q_{1}\right)$ to prepare the system in a stationary state, we can use it to compute the transition rate $k_{\mathrm{AB}}$ using equation (34):

$$
k_{\mathrm{AB}}=\frac{1}{\varepsilon_{\mathrm{A}}\left\langle n_{\mathrm{A}}\right\rangle_{\mathrm{eq}}}\left\langle\dot{q}_{1} \varepsilon\left(q_{1}\right) n_{\mathrm{A}}(t)\right\rangle_{\mathrm{eq}},
$$

where we have also taken into account that $\varepsilon\left(q_{1}\right) / \varepsilon_{\mathrm{A}}$ is approximately equal to $n_{\mathrm{A}}$ except in the barrier region, that contributes negligibly to equilibrium averages. From equation (136)

$$
\varepsilon\left(q_{1}\right)=-\varepsilon_{\mathrm{A}} \frac{\mathrm{e}^{\beta F_{e s t}\left(q_{1}\right)}}{\int_{q_{1 \mathrm{~A}}}^{q_{1 \mathrm{~B}}} \mathrm{~d} q_{\mathcal{1}} \mathrm{e}^{\beta F_{e s t}\left(q_{1}\right)}} .
$$

Let us define $p\left(q_{1}\right)$ as

$$
p\left(q_{1}\right)=\frac{\mathrm{e}^{\beta F_{\text {est }}\left(q_{1}\right)}}{\int_{q_{1 \mathrm{~A}}}^{q_{\mathrm{B}}} \mathrm{d} q_{\Gamma} \mathrm{e}^{\beta F_{\text {est }}\left(q_{1}\right)}} .
$$

Then, equation (137) can be rewritten as

$$
k_{\mathrm{AB}}=\frac{\left\langle\dot{q}_{1} p\left(q_{1}\right) n_{\mathrm{B}}(t)\right\rangle_{\mathrm{eq}}}{\left\langle p\left(q_{1}\right)\right\rangle_{\mathrm{eq}}} \frac{\left\langle p\left(q_{1}\right)\right\rangle_{\mathrm{eq}}}{\left\langle n_{\mathrm{A}}\right\rangle_{\mathrm{eq}}} .
$$

The first factor in this equation can be understood as a biased average, i.e., the average of $\dot{q}_{1} n_{\mathrm{B}}(t)$ with the initial distribution $\rho_{\text {eq }} p\left(q_{1}\right)$. If we denote this biased average by $\langle\ldots\rangle_{p}$, the final result we obtain for the transition rate is

$$
k_{\mathrm{AB}}=\left\langle\dot{q}_{1} n_{\mathrm{B}}(t)\right\rangle_{p} \frac{\left\langle p\left(q_{1}\right)\right\rangle_{\mathrm{eq}}}{\left\langle n_{\mathrm{A}}\right\rangle_{\mathrm{eq}}} .
$$

This equation has a very similar form to the usual expression, equation (39), for the rate, but now the constraining term is not a $\delta$ function, but a function that has the width of the barrier. We recall that, in practice, we approximate $F\left(q_{1}\right)$ by $F_{\text {est }}\left(q_{1}\right)$. But this is not really important: any reasonable guess for $p\left(q_{1}\right)$ will $\mathrm{do}$, and is bound to lead to a more rapid convergence that the $\delta$ function, as the perturbation that follows from it will be closer to the stationary state than the step function.

Still, equation (141) is not the best starting point for a numerical simulation. The biasing in equation (141) is such that it will compensate approximately the effect of the free energy barrier. Hence, the starting points for the trajectories of the simulation will be distributed almost uniformly over the entire range of $q_{1}$. In fact, we should expect that only trajectories starting in the barrier region yield relevant information. In order to see this we now use a characteristic function $n_{\mathrm{A}}$ that is the same as the initial perturbation, i.e., $n_{\mathrm{A}}=\varepsilon\left(q_{1}\right) / \varepsilon_{\mathrm{A}}$. 
When measuring the progress of the reaction we need to compute the change of $n_{\mathrm{A}}$ in an interval $t$. It now follows from equation (138) that

$$
n_{\mathrm{A}}(t)-n_{\mathrm{A}}(0)=-c \int_{0}^{\mathrm{d}} \mathrm{d} t^{\prime} \exp \left[\beta F_{\mathrm{est}}\left(q_{1}\left(t^{\prime}\right)\right)\right] \dot{1}_{1}\left(t^{\prime}\right)
$$

where we have denoted the normalization factor of equation (138) by $c$. Equation (142) shows clearly that only the barrier region, where $\exp \left(\beta F_{\text {est }}\left(q_{1}\right)\right)$ is large, contributes appreciably to the crossing rate. It is therefore convenient to write $n_{\mathrm{A}}(t)-n_{\mathrm{A}}(0)$ as

$$
\begin{aligned}
n_{\mathrm{A}}(t)-n_{\mathrm{A}}(0)= & -\exp \left[\beta F_{\text {est }}\left(q_{1}(0)\right)\right] \\
& \times \int_{0} \mathrm{~d} t^{\prime} \exp \left[\beta\left(F_{\text {est }}\left(q_{1}\left(t^{\prime}\right)\right)-F_{\text {est }}\left(q_{1}(0)\right)\right)\right] \\
& \times \dot{q}_{1}\left(t^{\prime}\right) .
\end{aligned}
$$

Rather than biasing our sampling with $p\left(q_{1}\right)$ defined in equation (139), we now define another weighting function $w\left(q_{1}\right)$ as

$$
w\left(q_{1}\right)=\frac{\mathrm{e}^{2 \beta F_{\text {est }}\left(q_{1}\right)}}{\int_{q_{1 \mathrm{~A}}}^{q_{1 \mathrm{~B}}} \mathrm{~d} q_{1} \mathrm{e}^{2 \beta F_{\text {est }}\left(q_{1}\right)}} .
$$

In exactly the same way as before, we now obtain the following expression for $k_{\mathrm{AB}}$

$$
\begin{aligned}
k_{\mathrm{AB}}= & \frac{\int_{q_{1 \mathrm{~A}}}^{q_{\mathrm{B}}} \mathrm{d} q_{1} \mathrm{e}^{2 \beta F_{\text {est }}\left(q_{1}^{\prime}\right)}}{\left[\int_{q_{1 \mathrm{~A}}}^{q_{1 \mathrm{~B}}} \mathrm{~d} q_{1} \mathrm{e}^{\beta F_{\text {est }}\left(q_{1}\right)}\right)^{2}} \int_{0} \mathrm{~d} t^{\prime} \\
& \times\left\langle\dot{q}_{1} \exp \left[\beta\left(F_{\text {est }}\left(q_{1}\left(t^{\prime}\right)\right)-F_{\text {est }}\left(q_{1}(0)\right)\right)\right] \dot{1}_{1}\left(t^{\prime}\right)\right\rangle_{w} \\
& \times \frac{\left\langle w\left(q_{1}\right)\right\rangle_{\mathrm{eq}}}{\left\langle n_{\mathrm{A}}\right\rangle_{\mathrm{eq}}} .
\end{aligned}
$$

Note that with $w$ as a biasing function, the sampling is indeed limited to the barrier region, so all the trajectories considered will contribute appreciably to the rate. In fact, the shape of the resulting distribution is proportional to $\exp \left(\beta\left[2 F_{\text {est }}\left(q_{1}\right)-F\left(q_{1}\right)\right] \approx \exp \left(\beta F\left(q_{1}\right)\right)\right.$. If the velocity $\dot{q}$ decays rapidly, then the factor $\exp \left[\beta\left(F_{\text {est }}\left(q_{1}\left(t^{\prime}\right)\right)-F_{\text {est }}\left(q_{1}(0)\right)\right)\right]$ will remain close to one.

We stress, once again, that accurate knowledge of $F\left(q_{1}\right)$, although useful when it is available, is not essential. Any reasonable approximation for $F\left(q_{1}\right)$ will, when inserted in equation (145), lead to improved statistical accuracy in our estimate of the transmission coefficient $\kappa$.

We should point out that several authors have considered the problem of diffusive barrier crossings from another perspective. Straub and Berne [19] devised a time-saving scheme to compute diffusive barriercrossing rates, based on the assumption that the dynamics of the trajectories before and after crossing the transition state are uncorrelated. Using this assumption, Straub and Berne then derive an expression for the transmission coefficient in terms of only those trajectories that remain on the product side for sufficiently long times. Therefore, only those trajectories are relevant and the simulation can be carried out with an absorbing boundary at the top of the barrier. Although this scheme is indeed cheaper than the original Bennett-Chandler method, it still suffers from transient effects. Moreover, as pointed out by Straub et al. [20] and by Gertner et al. [21] the approximations underlying this scheme may fail, even in the high friction limit. Borkovec and Talkner [22] addressed the problem of suppressing the transients in the computation of rate constants in the context of Markovian jump processes where $\dot{q}_{1}$ is ill defined. In our language, the approach of [22] is equivalent to preparing the system in a steady state. However, the characteristic function to measure the progress of the reaction is still the step function. As a consequence, the technique of [22] does not result in noise reduction. However, it should be emphasized that noise reduction was not the aim of [22]

\section{Discussion}

At this stage, the reader may well wonder why we do not simply use the Kramers equation to compute the transition rate. The point is that we use the Kramers picture only to arrive at a reasonable estimate for the function $\varepsilon\left(q_{1}\right)$. However, the validity of the rate expression that we derive does not assume that the Kramers equation is valid. In particular, the coordinate $q_{1}$ may depend in a nonlinear fashion on the Cartesian coordinates, in which case the simple diffusion equation will not hold. Moreover, the diffusion constant $D$ may depend on $q_{1}$ (if it is at all meaningful to define a local diffusion constant). Anyway, even if the barrier crossing is diffusive it may be described poorly by a FokkerPlanck equation. For instance, hydrodynamics effects that are not accounted for in the Fokker-Planck equation may be important for barrier crossing in solutions.

In summary, when computing rate constants of activated processes that exhibit appreciable recrossing, it is convenient to use an initial perturbation and characteristic functions that resemble the steady state concentration profiles. This is shown in figure 2, where we compare two different calculations of the barrier crossing rate for a labelled particle in a Lennard-Jones fluid. Only the labelled particle experiences an external potential shown in figure 1 . The dashed curve corresponds to the case when we use the conventional $\theta$ function perturbation and characteristic function. In this calculation, the system is initially constrained at the top of the barrier, and is then released. The numerical calculation of $k_{\mathrm{AB}}$ 


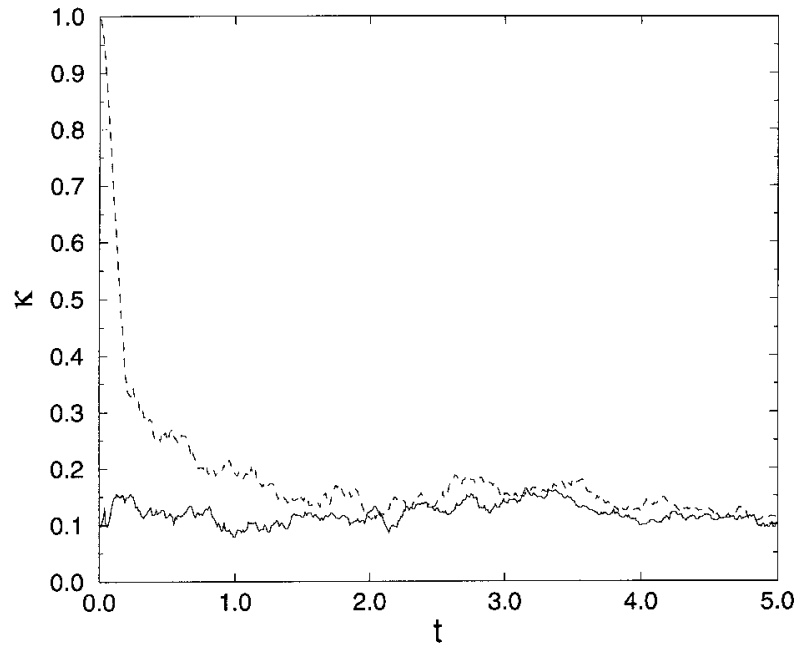

Figure 2. Two different calculations of the transition rate for the example discussed in the text. The dashed line corresponds to a $\theta$ shaped initial perturbation. The continuous line corresponds to an initial perturbation close to the steady state. The characteristic functions were $\theta$ functions in both cases.

shows two problems: it has a strong transient effect and the statistical noise in the plateau value is large. In fact, the magnitude of the statistical noise cannot be estimated simply by looking at the fluctuations in $k_{\mathrm{AB}}(t)$ as a function of time, because different points in this curve are strongly correlated. Rather, one should estimate the error by comparing the results obtained from a number of independent trajectory calculations [23] The dashed curve in figure 2 shows the result for $\kappa$ obtained using an initial perturbation that is close to the steady state profile for a purely diffusive barrier crossing process. We stress again that we do not assume that the Kramers picture is actually the correct description, only that it is a reasonable initial guess. Now we see that the transient effect in $\kappa$ is strongly suppressed. However, the statistical noise (due to the fact that we measure the progress of the reaction with a $\theta$ characteristic function) is still large. This can be seen in figure 3, where the continuous curve represents the same calculation of $k_{\mathrm{AB}}(t)$ as in figure 2 together with the estimated error in the plateau value. We find $\kappa=0.118 \pm 4.5 \times 10^{-2}$. The curve labelled by filled circles in the same figure shows the effect of using both the optimized initial perturbation and the appropriate characteristic function. This form is computationally more convenient as it leads to strong suppression of the transient behaviour and appreciable reduction in statistical noise. As expected, the estimate of $\kappa\left(\kappa=9.1 \times 10^{-2} \pm 1.7 \times 10^{-2}\right)$ is, to within statistical accuracy, the same as before. However, in the present case, the noise in $\kappa$ is reduced

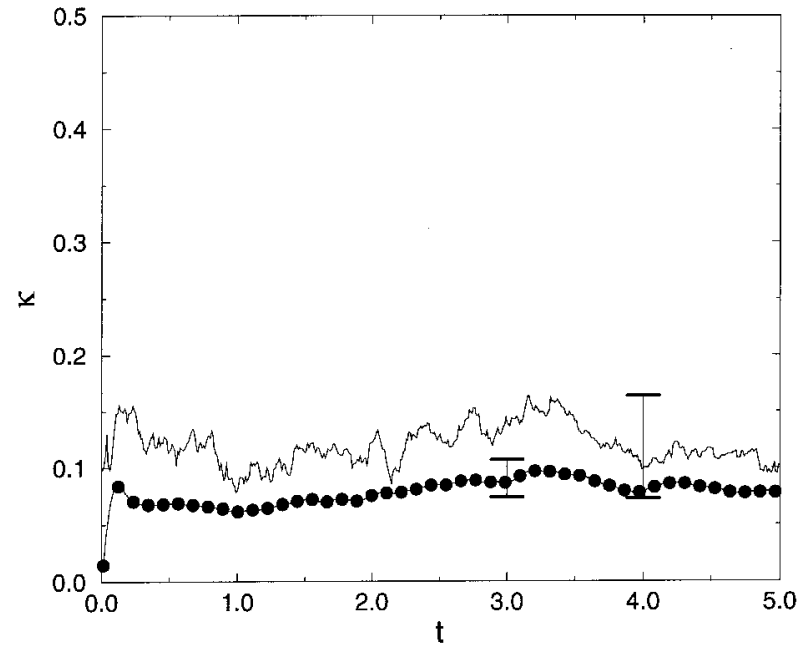

Figure 3. Comparison of two computations of the transition rate and its statistical noise when the system is prepared close to the steady state. The solid line corresponds to a $\theta$ characteristic function, and the line with circles to an optimized one.

by a factor of 3 (which is equivalent to almost a factor of 10 in computer time).

We stress that the example that we show here is by no means the best case as the barrier is rather narrow (around $2 \sigma$ ). For broader barriers, the computational gain would be much larger.

The work of the FOM Institute is part of the research program of the 'Stichting voor Fundamenteel Onderzoek der Materie' (FOM) and is supported by the Netherlands Organization for Scientific Research (NWO). Part of the computation reported in this work was supported through NWO grant MPR94.2186. M. J. R. acknowledges partial support from EU-HCM Grant Nr. ERBCHWICT941060. J. J. B. and M. J. R.-M. acknowledge Grant No. PB96-0534 from Dirección General de Investigación Cientifica y Técnica (Spain). We thank Giovanni Ciccotti and Pieter Rein ten Wolde for a critical reading of the manuscript, and David Chandler for bringing reference [22] to our attention.

\section{Appendix A}

\section{Phenomenological rate equation}

Let us suppose that the behaviour of $P_{\mathrm{A}}(t)$ and $P_{\mathrm{B}}(t)$, the probabilities of finding the system in states $\mathrm{A}$ and $\mathrm{B}$, is governed by the phenomenological equation

$$
\begin{aligned}
& \frac{\mathrm{d} P_{\mathrm{A}}(t)}{\mathrm{d} t}=-k_{\mathrm{AB}} P_{\mathrm{A}}(t)+k_{\mathrm{BA}} P_{\mathrm{B}}(t), \\
& \frac{\mathrm{d} P_{\mathrm{B}}(t)}{\mathrm{d} t}=k_{\mathrm{AB}} P_{\mathrm{A}}(t)-k_{\mathrm{BA}} P_{\mathrm{B}}(t),
\end{aligned}
$$


where $k_{\mathrm{AB}}$ and $k_{\mathrm{BA}}$ are constants. Of course, these equations verify the conservation condition

$$
\frac{\mathrm{d}}{\mathrm{d} t}\left(P_{\mathrm{A}}(t)+P_{\mathrm{B}}(t)\right)=0
$$

The equilibrium distribution has to be a stationary solution of the equation. Therefore,

$$
\frac{\mathrm{d} P_{\mathrm{A}}^{\mathrm{eq}}}{\mathrm{d} t}=-k_{\mathrm{AB}} P_{\mathrm{A}}^{\mathrm{eq}}+k_{\mathrm{BA}} P_{\mathrm{B}}^{\mathrm{eq}}=0,
$$

which implies

$$
\frac{k_{\mathrm{AB}}}{k_{\mathrm{BA}}}=\frac{P_{\mathrm{B}}^{\mathrm{eq}}}{P_{\mathrm{A}}^{\mathrm{eq}}} .
$$

Since $P_{\mathrm{A}}(t)+P_{\mathrm{B}}(t)=1$, we find for $\Delta P_{\mathrm{A}}(t)=$ $P_{\mathrm{A}}(t)-P_{\mathrm{A}}^{\mathrm{eq}}$ that

$$
\frac{\mathrm{d} \Delta P_{\mathrm{A}}}{\mathrm{d} t}=-\left(k_{\mathrm{AB}}+k_{\mathrm{BA}}\right) \Delta P_{\mathrm{A}}(t)
$$

It follows directly that the relaxation of the system is exponential:

$$
\Delta P_{\mathrm{A}}(t)=\Delta P_{\mathrm{A}}(0) \mathrm{e}^{-t / \tau}
$$

with

$$
\begin{aligned}
\tau^{-1} & =k_{\mathrm{AB}}+k_{\mathrm{BA}}=k_{\mathrm{AB}}\left(1+\frac{k_{\mathrm{BA}}}{k_{\mathrm{AB}}}\right) \\
& =k_{\mathrm{AB}}\left(1+\frac{P_{\mathrm{A}}^{\mathrm{eq}}}{P_{\mathrm{B}}^{\mathrm{eq}}}\right) \\
\tau^{-1} & =\frac{k_{\mathrm{AB}}}{P_{\mathrm{B}}^{\mathrm{eq}}}
\end{aligned}
$$

This is equation (33) in the main text.

\section{Appendix B}

Independence of $K_{A B}$ of location of barrier

We wish to investigate the dependence of the transition rate given by equation (39) on the choice of the transition state $q_{1}^{*}$. It is a well known result [6] that the TST expression for the rate does depend on the choice of $q_{1}^{*}$. Equation (39) for the rate is:

$$
k_{\mathrm{AB}}=\frac{\left\langle\dot{q}_{1} \delta\left(q_{1}-q_{1}^{*}\right) \theta\left[q_{1}(t)-q_{1}^{*}\right]_{\mathrm{eq}}\right.}{\left\langle\theta\left(q_{1}^{*}-q_{1}\right)\right\rangle_{\mathrm{eq}}} .
$$

First of all, it is clear that the RHS of equation (B 1) cannot be independent of $q_{1}^{*}$ for all times. For instance, we know that the limit of this equation for $t \rightarrow 0^{+}$is the TST prediction for the rate, and it depends on time. However, we are interested only in times such that the plateau of the previous expression has been established.

Let us consider the denominator of equation (B 1). It is the equilibrium probability of finding the system in state A. It is clear that, if we choose $q_{1}^{*}$ in the barrier region, where the probability of finding the system is very small, a small shift in its value will hardly affect the equilibrium probability of state A. Hence, we can consider the denominator of equation (B 1) to be independent on $q_{1}^{*}$ as long as it is located in the barrier region. The next step is to study the dependence of the numerator:

$$
\begin{aligned}
\left\langle\dot{q}_{1} \delta\left(q_{1}-q_{1}^{*}\right) \theta\left[q_{1}(t)-q_{1}^{*}\right)\right. & \rangle_{\mathrm{eq}} \\
& =\left\langle\mathscr{B} \theta\left(q_{1}-q_{1}^{*}\right) \mathrm{e}^{t \mathscr{B}} \theta\left(q_{1}-q_{1}^{*}\right)\right\rangle_{\mathrm{eq}} .
\end{aligned}
$$

Differentiating with respect to $q_{1}^{*}$ and using timereversal symmetry, we obtain

$$
\begin{aligned}
& \frac{\partial}{\partial q_{1}^{*}}\left\langle\dot{q}_{1} \delta\left(q_{1}-q_{1}^{*}\right) \theta\left[q_{1}(t)-q_{1}^{*}\right)\right\rangle_{\mathrm{eq}} \\
&=\left\langle\frac{\partial \theta\left(q_{1}-q_{1}^{*}\right)}{\partial q_{1}^{*}} \mathrm{e}^{t \mathscr{S}} \theta\left(q_{1}-q_{1}^{*}\right)\right\rangle_{\mathrm{eq}} \\
&+\left\langle\mathscr{L} \theta\left(q_{1}-q_{1}^{*}\right) \mathrm{e}^{t \mathscr{S}} \frac{\partial \theta\left(q_{1}-q_{1}^{*}\right)}{\partial q_{1}^{*}}\right\rangle_{\mathrm{eq}} \\
&=-\left\langle\frac{\partial \theta\left(q_{1}-q_{1}^{*}\right)}{\partial q_{1}^{*}} \mathrm{e}^{t \mathscr{E}} \mathscr{B} \theta\left(q_{1}-q_{1}^{*}\right)\right\rangle_{\mathrm{eq}} \\
&-\left\langle\frac{\partial \theta\left(q_{1}-q_{1}^{*}\right)}{\partial q_{1}^{*}} \mathrm{e}^{t \mathscr{S}} \mathscr{B} \theta\left(q_{1}-q_{1}^{*}\right)\right\rangle_{\mathrm{eq}} \\
&= 2\left\langle\delta\left(q_{1}-q_{1}^{*}\right) \dot{q}_{1}(t) \delta\left(q_{1}(t)-q_{1}^{*}\right)\right\rangle_{\mathrm{eq}} \cdot
\end{aligned}
$$

This is the velocity of the system at time $t$, multiplied by the probability that it is at the top of the barrier at time $t$ and also was at the top of the barrier at time $t=0$. There is no symmetry reason why this correlation function should vanish. In fact, for sufficiently short times it is clear that it is different from zero. For these times, the derivative of $k_{\mathrm{AB}}$ with respect to $q_{1}^{*}$ is non-zero, and the rate depends on the choice of $q_{1}^{*}$. On the other hand, for times long enough for this correlation function to have decayed, the transition rate will be independent of the precise choice of the transition state, as long as it is chosen in the barrier region.

\section{Appendix C}

Relation between $\mathscr{B}$ and $\mathscr{G}$

The inverse of the matrix $\mathscr{G}$ defined in equation (51) is given by

$$
(\mathscr{G})_{\alpha \beta}^{-1}=\sum^{N} m_{i}^{-1} \frac{\partial q_{\alpha}}{\partial \mathbf{r}_{i}} \cdot \frac{\partial q_{\beta}}{\partial \mathbf{r}_{i}},
$$

as can be verified by using

$$
\sum_{\alpha}^{3 N} \frac{\partial \mathbf{r}_{i}}{\partial q_{\alpha}} \frac{\partial q_{\alpha}}{\partial \mathbf{r}_{j}}=\delta_{i j} \mathbf{I}
$$


where $\mathbf{I}$ is the $3 \times 3$ unit tensor. Let us write the matrices $\mathscr{G}$ and $\mathscr{G}^{-1}$ in block form as

$$
\mathscr{G}=\left(\begin{array}{cc}
\mathscr{G}_{11} & \mathscr{G}_{1 \mathrm{~s}} \\
\mathscr{G}_{\mathrm{s} 1} & \mathscr{G}_{\mathrm{s}}
\end{array}\right), \quad \mathscr{G}^{-1}=\left(\begin{array}{cc}
H & B_{1 \mathrm{~s}} \\
B_{\mathrm{s} 1} & B_{\mathrm{s}}
\end{array}\right),
$$

where

$$
H=(\mathscr{G})_{11}^{-1}=\sum_{4}^{N} m_{i}^{-1} \frac{\partial q_{1}}{\partial \mathbf{r}_{i}} \cdot \frac{\partial q_{1}}{\partial \mathbf{r}_{i}} .
$$

We also define a matrix $X$ by

$$
X=\left(\begin{array}{cc}
1 & \mathscr{G}_{1 \mathrm{~s}} \\
0 & \mathscr{G}_{\mathrm{s}}
\end{array}\right) .
$$

By construction this matrix has the same determinant as $\mathscr{G}_{\mathrm{s}}$,

$$
|X|=\left|\mathscr{G}_{\mathrm{s}}\right| \text {. }
$$

The relation $\mathscr{G}^{-1} \mathscr{G}=I$ implies

$$
\begin{aligned}
& H \mathscr{G}_{1 \mathrm{~s}}+B_{1 \mathrm{~s}} \mathscr{G}_{\mathrm{s}}=0, \\
& B_{\mathrm{s} 1} \mathscr{G}_{1 \mathrm{~s}}+B_{\mathrm{s}} \mathscr{G}_{\mathrm{s}}=I_{\mathrm{s}},
\end{aligned}
$$

and use of this leads to

$$
\mathscr{G}^{-1} X=\left(\begin{array}{cc}
H & 0 \\
B_{\mathrm{s} 1} & I_{\mathrm{s}}
\end{array}\right)
$$

and therefore

$$
\left|\mathscr{G}^{-1} X\right|=H
$$

It then follows that

$$
\left|\mathscr{G} \mathscr{G}^{-1} X\right|=|\mathscr{G}|\left|\mathscr{G}^{-1} X\right| \mathscr{G}|| H \mid,
$$

and also that

$$
\left|\mathscr{G G}^{-1} X\right|=|X|=\left|\mathscr{G}_{\mathrm{s}}\right| .
$$

Comparison of equations (C10) and (C11) leads to equation (62) in the main text.

\section{Appendix D}

Simplifying Jacobians

In order to make the notation in this appendix more transparent we rename the Cartesian coordinates as

$$
\left\{\mathbf{r}_{1}, \mathbf{r}_{2}, \ldots, \mathbf{r}_{N}\right\} \equiv\left\{r_{\alpha} ; \alpha=1, \ldots, 3 N\right\} .
$$

The matrix associated to the transformation from Cartesian to generalized coordinates is

$$
J_{\alpha \beta}=\frac{\partial r_{\alpha}}{\partial q_{\beta}}
$$

and its inverse is

$$
\left(J^{-1}\right)_{\alpha \beta}=\frac{\partial q_{\alpha}}{\partial r_{\beta}}
$$

Denoting the transpose of $J$ by $J^{+}$, we introduce a matrix $Q$ defined as

$$
\begin{aligned}
Q_{\alpha \beta} \equiv\left[J^{-1}\left(J^{-1}\right)^{+}\right]_{\alpha \beta} & =\sum_{\mu} \frac{\partial q_{\alpha}}{\partial r_{\gamma}} \frac{\partial q_{\beta}}{\partial r_{\gamma}} \\
& =\nabla q_{\alpha} \cdot \nabla q_{\beta}=\left|\nabla q_{\alpha}\right|^{2} \delta_{\alpha, \beta} .
\end{aligned}
$$

In the last transformation we employed equations (83) and (84) from the main text. Note that $Q$ is a diagonal matrix with all the diagonal elements equal to unity except the first one. Therefore, $\left(Q^{-1}\right)_{\alpha \beta}=\left|\nabla q_{\alpha}\right|^{-2} \delta_{\alpha \beta}$. This will be used below. Moreover,

$$
\left|J^{-1}\left(J^{-1}\right)^{+}\right|=|J|^{-2}=\left|\nabla q_{1}\right|^{2}
$$

and

$$
|J|=\left|\nabla q_{1}\right|^{-1} .
$$

Let us now consider an arbitrary function of the position of the particles $f\left(\mathbf{r}_{1}, \mathbf{r}_{2}, \ldots, \mathbf{r}_{N}\right)$. The derivative of this function with respect to the reaction coordinate, keeping all the other generalized coordinates constant, is given by

$$
\frac{\partial f}{\partial q_{1}}=\sum_{\mu}^{3 N} \frac{\partial f}{\partial r_{\alpha}} \frac{\partial r_{\alpha}}{\partial q_{1}}
$$

In order to get an expression for $J_{\alpha 1} \equiv \partial r_{\alpha} / \partial q_{1}$ let us consider

i.e.,

$$
J=\left(J^{-1}\right)^{+} Q^{-1}
$$

$$
J_{\alpha \beta}=\sum_{\psi}^{3 N} \frac{\partial q_{\gamma}}{\partial r_{\alpha}}\left(Q^{-1}\right)_{\gamma \beta}=\frac{1}{\left|\nabla q_{\beta}\right|^{2}} \frac{\partial q_{\beta}}{\partial r_{\alpha}} .
$$

Using this result in equation (D 7) we finally obtain

$$
\frac{\partial f}{\partial q_{1}}=\frac{1}{\left|\nabla q_{1}\right|^{2}} \sum_{\mathbf{y}}^{3 N} \frac{\partial f}{\partial r_{\alpha}} \frac{\partial q_{1}}{\partial r_{\alpha}}=\frac{\nabla f \cdot \nabla q_{1}}{\left|\nabla q_{1}\right|^{2}},
$$

which is equation (87).

\section{References}

[1] Proceedings of the Symposium on Rate Processes and First Passage Times, 1986, J. statist. Phys., 42.

[2] Hänggi, P., Talkner, P., and Borkovec, M., 1990, Rev. mod. Phys., 62, 251.

[3] Cic cotti, G., Ferrario, M., Hynes, J. T., and Kapral, R., 1989, Chem. Phys., 129, 241; 1990, J. chem. Phys., 93, 7137.

[4] Bennett, C. H., 1975, Diffusion in Solids: Recent Developments, edited by J. J. Burton and A. S. Norwick (New York: Academic Press).

[5] van Du ijneveldt, J. S., and Frenkel, D., 1990, J. chem. Phys., 96, 4655; ten Wolde, T. R., Ru iz-Montero, M. J., and Frenkel, D., 1995, Phys. Rev. Lett., 75, 2714.

[6] Chandler, D., 1978, J. chem. Phys., 68, 2959; 1987, Introduction to Modern Statistical Mechanics (Oxford University Press).

[7] Kramers, H. A., 1940, Physica, 7, 284. 
[8] Carter, E. A., Ciccotti, G., Hynes, J. T., and Kapral, R., 1989, Chem. Phys. Lett., 156, 472; Cicсотті, G., 1991, Computer Simulations in Materials Science, edited by M. Meyer and V. Pontikis (Dordrecht: Kluwer), p. 119.

[9] Luzar, A., and Chandler, D., 1997, Phys. Rev. Lett., 76, 928 .

[10] Miller, W. H., 1974, J. chem. Phys., 61, 1823; 1976, Accounts chem. Res., 9, 306.

[11] Glasstone, S., Laidler, K. J., and Eyring, H., 1941, Theory of Rate Processes (New York: McGraw-Hill).

[12] Goldstein, H., 1980, Classical Mechanics, 2nd Edn (Reading, MA: Addison-Wesley).

[13] Torrie, G. M., and Valleau, J. P., 1977, J. comput. Phys., 23, 187.

[14] ten Wolde, P. R., Ruiz -Montero, M. J., and Frenkel, D., 1997, J. chem. Phys., in press.
[15] Allen, M. P., and Tildesley, D. J., 1987, Computer Simulation of Liquids (Oxford: Clarendon Press).

[16] Skinner, J. L., and Wolynes, P. G., 1980, J. chem. Phys., 72, 4913.

[17] van Ka mpen, N. G., 1977, J. statist. Phys., 17, 71.

[18] Risken, H., 1984, The Fokker-Planck Equation (Berlin: Springer-Verlag), p. 114.

[19] Straub, J. E., and Berne, B. J., 1985, J. chem. Phys., 83, 1138.

[20] Straub, J. E., Hsu, D. A., and Berne, B. J., 1985, J. phys. Chem., 89, 5188.

[21] Gertner, B. J., Wilson, K. R., and Hynes, J. T., 1989, J. chem. Phys., 90, 3537.

[22] Borkovec, M., and Ta lkner, P., 1990, J. chem. Phys., 92, 5307.

[23] Rey, R., 1996, J. chem. Phys., 104, 1966. 
